\title{
Open, closed, and shared access femtocells in the downlink
}

\author{
Han-Shin Jo ${ }^{1 *}$, Ping Xia ${ }^{2}$ and Jeffrey G Andrews ${ }^{2}$
}

\begin{abstract}
A fundamental choice in femtocell deployments is the set of users which are allowed to access each femtocell. Closed access restricts the set to specifically registered users, while open access allows any mobile subscriber to use any femtocell. Which one is preferable depends strongly on the distance between the macrocell base station (MBS) and femtocell. The main results of the this article are lemmas which provide expressions for the signal-to-interference-plus-to-noise ratio (SINR) distribution for various zones within a cell as a function of this MBS-femto distance. The average sum throughput (or any other SINR-based metric) of home users and cellular users under open and closed access can readily be determined from these expressions. We show that unlike in the uplink, the interests of home and cellular users are in conflict, with home users preferring closed access and cellular users preferring open access. The conflict is most pronounced for femtocells near the cell edge, when there are many cellular users and fewer femtocells. To mitigate this conflict, we propose a middle way which we term shared access in which femtocells allocate an adjustable number of time-slots between home and cellular users such that a specified minimum rate for each can be achieved. The optimal such sharing fraction is derived. Analysis shows that shared access achieves at least the overall throughput of open access while also satisfying rate requirements, while closed access fails for cellular users and open access fails for the home user.
\end{abstract}

\section{Introduction}

Femtocells are small form-factor base stations that can be installed within an existing cellular network. They can be installed either by an end-user or by the service provider and are distinguished from pico or microcells by their low cost and power and use of basic IP backhaul, and from WiFi by their use of cellular standards and licensed spectrum [1]. Femtocells are a very promising and scalable method for meeting the ever-increasing demands for capacity and high-rate coverage. Since femtocells share spectrum with macrocell networks, managing cross-tier interference between femto- and macrocells is essential [2-4]. Furthermore, a basic question, particularly for enduser-installed femtocells, is which users in the network should be allowed to use a given femtocell.

\subsection{Motivation and related work}

Cross-tier interference is highly dependent on this femtocell access decision. Closed access, where only specified

\footnotetext{
*Correspondence: hsjo@hanbat.ac.kr

1 Department of Electronics and Control Engineering, National Hanbat University, Daejeon, Korea

Full list of author information is available at the end of the article
}

registered home users can communicate with the femtocell access point (FAP), appears attractive to the home user but can result in severe cross-tier interference from nearby cellular users in the uplink (see Figure 1) or to nearby cellular users in the downlink. To reduce this interference in closed access, previous studies have considered power control [5-9], frequency assignment [10-12], and a spectrum sensing approach $[13,14]$. An alternative is to simply hand over cellular users that cause or experience strong interference to the femtocell. This is known as open access. Intuitively, this should increase the overall network capacity [15] at the possible expense of a given femtocell owner, who must now share his femtocell resources (time/frequency slots and backhaul) with an unpredictable number of cellular users.

The uplink performance of femtocell access schemes has been investigated in $[16,17]$. The interrelationship between the traffic type, access policy, and performance of high-speed packet access (HSPA) was examined in a simulation-based study [16]. In [17], an analytical framework was presented from open versus closed access. Both studies suggested a hybrid access approach with an upper limit to the number of unregistered users to access the

\section{黛 Springer}




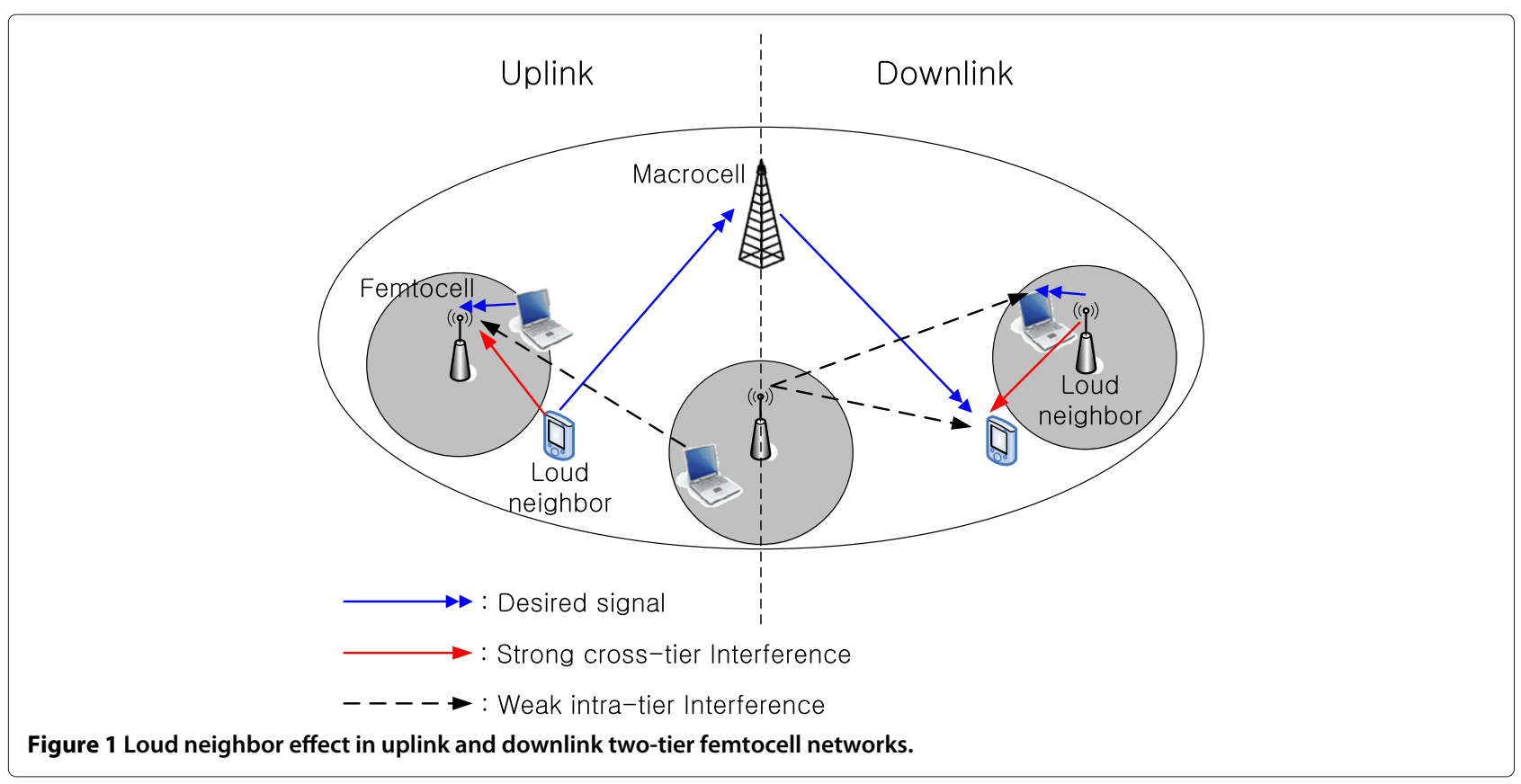

femtocell. We term this approach shared access since the femtocell is shared with cellular users, but within limits and hence not fully open. With respect to the uplink throughput of registered home users, open (or shared) access reduces interference by handing over the loud neighbor at the expense of FAP resource sharing. The tradeoff is such that open access is generally preferred for both home users and cellular users, since the interference reduction is so important [17]. Does the same tradeoff hold in the downlink?

It would seem that the tradeoff is different in the downlink since here the FAP is the loud neighbor (see Figure 1). Therefore, serving unregistered users with the FAP benefits them at the cost of FAP resources. Meanwhile, there is at best a very small decrease in downlink interference to the home user. The downlink capacity of open versus closed access has been studied using simulations for HSPA femtocells [16,18] and OFDMA femtocells [19-21]. These studies propose and analyze shared access methods with limits on the number of unregistered users [18] and frequency subchannels for them [20,21]. Indeed, these studies find that cellular user performance is improved with open (or shared) access at the cost of reduced home user performance.

Recently, a theoretical analysis of signal-to-interferenceplus-to-noise ratio (SINR) in general $K$-tier downlink heterogeneous cellular networks with open or closed access was proposed in [22-24]. For $K=2$, macrocells plus femtocells can be considered as a special case. Analytical results on downlink outage probability for open and closed access have also been been presented in [25]. However, these models do not classify mobile users into femtocell-registered (home users) and femtocellunregistered (cellular users), and does not define the distribution area of each type of users. It is thus hard to quantify the throughput of the registered home users and unregistered cellular users separately. Furthermore, since all BSs are distributed as a Poisson Point Process (PPP), the feasible distance between macrocell base station (MBS) and femtocell is arbitrarily given within a range from zero to infinity (i.e., not fixed). Such a random BS model appears useful for obtaining outage/throughput averaged over the entire network, but may not quantify the throughput of open, closed, and shared access for a particular femtocell at a given distance from the MBS.

\subsection{Contributions and main insights}

Clearly, it is desirable to quantify the throughput of open, closed, and shared access as a function of the FAP-MBS distance. It should provide the best access or optimal resource allocation (in shared access) for each femtocell based on its location, and even the density of femtocells and cellular users. It would be more realistic if the femtocell and cellular user positions were not fixed, but rather were modeled as a spatial random process (see $[26,27]$ and references therein). Unlike the closed access model $[9,28]$ with a fixed radio range (coverage) for the femtocell, we develop a unified model for both open and closed accesses, where the femtocell coverage area depends on its distance from the MBS. In addition, the femtocell coverage is divided into zones, each of which has unique SINR model due to path loss and wall loss (indoor/outdoor), type of serving BS (MBS/FAP), and access control (open/closed). Ideally, a general statistical 
distribution of the SINR for the various zones could be found. Since metrics such as outage probability, error probability, and throughput follow directly from SINR, once the SINR distribution is known these metrics can be computed quite quickly and easily [27]. Deriving such an SIR distribution (we neglect both thermal noise and interference from other cells) is the main contribution of this article, and is used to draw a few conclusions about access strategies in the downlink.

First, we see that unlike the uplink [17], the preferred access schemes for home and cellular users are incompatible, with home user preferring closed access. For femtocells with coverage area extending outside the home, i.e., far from the MBS, closed access provides higher sum throughput for home user and lower sum throughput for neighboring cellular users, when compared to open access. For example, in a cell edge femtocell, open access causes at least $20 \%$ throughput loss to home user compared to closed access, while the neighboring cellular user experiences outage for typical data service (less than $15 \mathrm{kbps}$ for $5 \mathrm{MHz}$ bandwidth) in closed access. Furthermore, we observe that the open access femtocells far from MBS reduce the macrocell load, thereby open access rather than closed access offers higher throughput for a few home users (in its femtocell coverage area smaller than home area) located near and connected to the MBS. Nevertheless, most home users in cell site accessing FAPs are still reluctant to use their femtocells in open access.

Since neither open nor closed access can completely satisfy the need of both user groups, we next propose a shared access approach where the femtocell has a timeslot ratio $\eta$ between the home and cellular users it serves, where $\eta=1$ is closed access. An optimal value of $\eta$ is found to maximize network throughput subject to QoS requirements on the minimum throughput per home and cellular user. For example, given a cell edge femtocell with minimum throughput of $50 \mathrm{kbps} /$ cellular user and $500 \mathrm{kbps} /$ home user, this shared access approach achieves about $80 \%$ higher network throughput than open access. When the QoS requirements increase in favor of significantly higher throughput of cellular user, shared access provides the lower network throughput than open access.

\section{System model}

We consider single macrocell modeled by a disc with radius $R_{\mathrm{C}}$ centered at an MBS. To show the impact of macro-femto distance on femtocell access control, we analyze a target femtocell, its location denoted as $X_{0}$, which is located at a distance $D$ away from the MBS. Since FAPs are installed by the end customer, they are distributed with randomness rather than regular pattern. FAPs are thus distributed according to a homogeneous $\operatorname{PPP} \Phi=\left\{X_{j}\right\}_{j=1,2, \ldots}$ with intensity $\lambda$, where each $X_{j}$ is the location of the $j$ th FAP. The mean number of femtocells per cell cite is given as $N_{\mathrm{f}}=\pi \lambda R_{\mathrm{c}}^{2}$. Home users are uniformly deployed indoors (home), a disc $\mathcal{H} \subset \mathbb{R}^{2}$ of radius $R_{i}$ centered at their FAP. Cellular users are uniformly distributed outdoors. A summary of notation is given in Table 1.

\subsection{Channel model and multi-level modulation}

The downlink channel experiences path loss, Rayleigh fading with unit average power, and wall penetration loss $L<$ 1 . The path loss exponents are denoted by $\alpha$ (outdoor and outdoor-to-indoor or indoor-to-outdoor) and $\beta$ (indoorindoor). As in $[9,12,30]$, the downlink femtocell networks are assumed to be interference-limited and thermal noise at the receiver is ignored for simplicity. The MBS and FAP use fixed ${ }^{\mathrm{a}}$ transmit powers of $P_{\mathrm{C}}$ and $P_{\mathrm{f}}$, respectively. We assume that orthogonal multiple access is used (TDMA or OFDMA on a per subband basis), thus no intracell interference is considered. Interference from neighboring macrocell BSs is ignored for analytical tractability. ${ }^{\text {b }}$ We consider multi-level $\mathrm{M}$-ary modulation single carrier transmission that is adapted to the received SIR $\gamma$, thus each user is assumed to estimate its SIR and provide perfect SIR feedback to their MBS (or FAP). Define $N$ SIR regions as $\mathcal{G}_{n}=\left[\Gamma_{n}, \Gamma_{n+1}\right), n=1, \ldots, N$, where $\Gamma_{1}$ is the minimum SIR providing the lowest discrete rate and $\Gamma_{N+1}=\infty$. Then, the instantaneous transmission rate (in $\mathrm{bps} / \mathrm{Hz}$ ) is

$$
r_{n}=\log _{2}\left(1+\frac{\Gamma_{n}}{G_{n}}\right) \text { for } \gamma \in \mathcal{G}_{n}, 1 \leq n \leq N,
$$

where $G_{n}$ is the Shannon Gap of M-ary modulation for the $n$th SIR region (and may assume some level of coding). Assuming round robin (RR) scheduling with equal time slots, the average throughput is

$$
T=\sum_{n=1}^{N} r_{n} \cdot \mathbb{P}\left[\Gamma_{n} \leq \gamma<\Gamma_{n+1}\right]
$$

\subsection{Femtocell coverage and cell association}

We assume a max-SINR cell association where each mobile users is served by the station (MBS or FAP) providing the strongest average power to them. This is desirable in that it maximizes the user SINRs in downlink. We therefore define a femtocell coverage $\mathcal{F} \subset \mathbb{R}^{2}$ as the area inside which the average received power from the FAP is stronger than that from the central MBS. The coverage is mathematically modeled by the following lemma. ${ }^{\mathrm{c}}$

Lemma 1. For an FAP at distance D from a central MBS located at the origin, the femtocell coverage area approximately confined to a circle centered at $(D, 0)$, and its radius $R_{\mathrm{f}}$ is

$$
R_{\mathrm{f}}=\kappa^{-1 / \alpha} D,
$$

where $\kappa=\frac{P_{\mathrm{C}}}{P_{\mathrm{f}} L} \gg 1$. 


\begin{tabular}{|c|c|c|}
\hline Symbol & Description & Sim. value \\
\hline $\mathcal{F}_{i}$ & Indoor area covered by the FAP at $D>D_{\mathrm{th}}$ (a disc with the radius $\left.R_{i}\right)$ & N/A \\
\hline \multirow[t]{3}{*}{$\mathcal{F}_{\circ}$} & Outdoor area covered by the FAP at $D>D_{\text {th }}$ in open access or & \\
\hline & covered by the MBS in closed access & N/A \\
\hline & (a circular annulus with inner radius $R_{\mathbf{i}}$ and outer radius $R_{\mathbf{f}}$ ) & \\
\hline $\mathcal{F}_{\mathrm{a}}$ & Indoor area covered by the FAP at $D \leq D_{\text {th }}$ (a disc with the radius $R_{\mathrm{f}}$ ) & N/A \\
\hline \multirow[t]{2}{*}{$\mathcal{F}_{\mathrm{b}}$} & Indoor area covered by the MBS (a circular annulus with inner radius $R_{\mathrm{f}}$ & N/A \\
\hline & and outer radius $R_{\mathrm{i}}$ with respect to the FAP at $D \leq D_{\mathrm{th}}$ ) & \\
\hline$D$ & Distance between FAP and central MBS & Not fixed \\
\hline$D_{\text {th }}$ & Threshold distance (Radius of inner region) & Not fixed \\
\hline$D_{c}$ & Distance between central MBS and homeuser (or neighboring cellular user) & Not fixed \\
\hline$R$ & Distance between FAP and homeuser (or neighboring cellular user) & Not fixed \\
\hline$R_{\mathrm{f}}$ & Femtocell radius & Not fixed \\
\hline$R_{\mathrm{c}}$ & Macrocell radius & $500 \mathrm{~m}$ \\
\hline$R_{\mathrm{i}}$ & Indoor (home) area radius & $20 \mathrm{~m}$ \\
\hline$P_{\mathrm{c}}$ & Transmit power at macrocell & $43 \mathrm{dBm}[29]$ \\
\hline$P_{\mathrm{f}}$ & Transmit power at femtocell & $13 \mathrm{dBm}[29]$ \\
\hline$\alpha$ & Outdoor path loss exponent & 4 \\
\hline$\beta$ & Indoor path loss exponent & 4 \\
\hline L & Wall penetration loss & $0.5(-3 \mathrm{~dB})$ \\
\hline$G_{n}$ & Shannon gap & $3 \mathrm{~dB}$ \\
\hline N & Number of discrete levels for M-ary modulation (M-QAM) & 8 \\
\hline$\Omega_{\mathrm{c}}$ & Required minimum throughput of cellular user for hybrid access & $0.01 \mathrm{bps} / \mathrm{Hz}$ \\
\hline$\Omega_{\mathrm{h}}$ & Required minimum throughput of home user for hybrid access & $0.1 \mathrm{bps} / \mathrm{Hz}$ \\
\hline
\end{tabular}

Proof. Consider a central MBS located at $(0,0)$ and an FAP at distance $D$ from the MBS. Without loss of generality, the FAP is assumed to be located at $(D, 0)$. The received power at the position $(x, y)$ with distances $D_{\mathrm{C}}=$ $\sqrt{x^{2}+y^{2}}$ and $R=\sqrt{(D-x)^{2}+y^{2}}$ from MBS and FAP are, respectively, given as $P_{r}^{(c)}=P_{\mathrm{C}} P_{0}\left(\frac{D_{\mathrm{c}}}{d_{0}}\right)^{-\alpha}$ and $P_{\Upsilon}^{(f)}=$ $P_{\mathrm{f}} L P_{0}\left(\frac{R}{d_{0}}\right)^{-\alpha}$, where $P_{0}$ is the path loss at a reference distance $d_{0}$. The contour with $P_{r}^{(c)}=P_{r}^{(f)}$ (zero dB SIR) satisfies $\frac{x^{2}+y^{2}}{(D-x)^{2}+y^{2}}=\kappa^{\alpha / 2}$, where $\kappa=\frac{P_{\mathrm{C}}}{P_{\mathrm{f}} L} \gg 1$. The equation is rewritten as $\left(x-\frac{\kappa^{2 / \alpha} D}{\kappa^{2 / \alpha}-1}\right)^{2}+y^{2}=\frac{\kappa^{2 / \alpha} D^{2}}{\left(\kappa^{2 / \alpha}-1\right)^{2}}$. Note that $\frac{\kappa^{2 / \alpha}}{\kappa^{2 / \alpha}-1}$ is very close to 1 because $\kappa \gg 1$. For example, $\frac{\kappa^{2 / \alpha}}{\kappa^{2 / \alpha}-1}=1.02$ for the values in Table 1. Thus, the equation is simplified to $(x-D)^{2}+y^{2}=\kappa^{-2 / \alpha} D^{2}$, which is the equation of circle and completes the proof.

Lemma 1 states that the FAP is located at the center of the femtocell coverage area, and that the femtocell coverage radius linearly increase with the distance $D$ from the MBS. This implies that for an FAP close to the MBS, femtocell coverage can be smaller than indoor area, whereas for an FAP far from the MBS, the coverage is larger than indoor area and leaks into outdoors as shown in Figure 2. We thus denote $D_{\text {th }}$ as a nominal threshold distance at which the femtocell coverage area is exactly equal to the indoor (home) area, i.e., $R_{\mathrm{f}}=R_{\mathrm{i}}$. We accordingly partition the macrocell into two regions, inner region $(D \leq$ $\left.D_{\text {th }}\right)$ and outer region $\left(D>D_{\text {th }}\right)$. Note that $D_{\text {th }}$ is the same for all FAPs, since we assume all indoor regions with same area.

For all types of femtocell access control, the home users can freely choose between the MBS and their own femtocell according to the association policy. Thus, they will be served by their own femtocell if in its coverage, and by the MBS otherwise. On the other hand, whether a cellular user will be served by a femtocell is more complicated and dependent on the type of access control: (1) in closed access, a femtocell will not serve any cellular users, even if they are in its coverage; (2) in the open access, a femtocell will serve any cellular users as long as they are in its coverage, they are named neighboring cellular users. In the inner region where the femtocell coverage area is smaller than the indoor area, some home users outside the femtocell coverage actually communicate with the MBS, while 


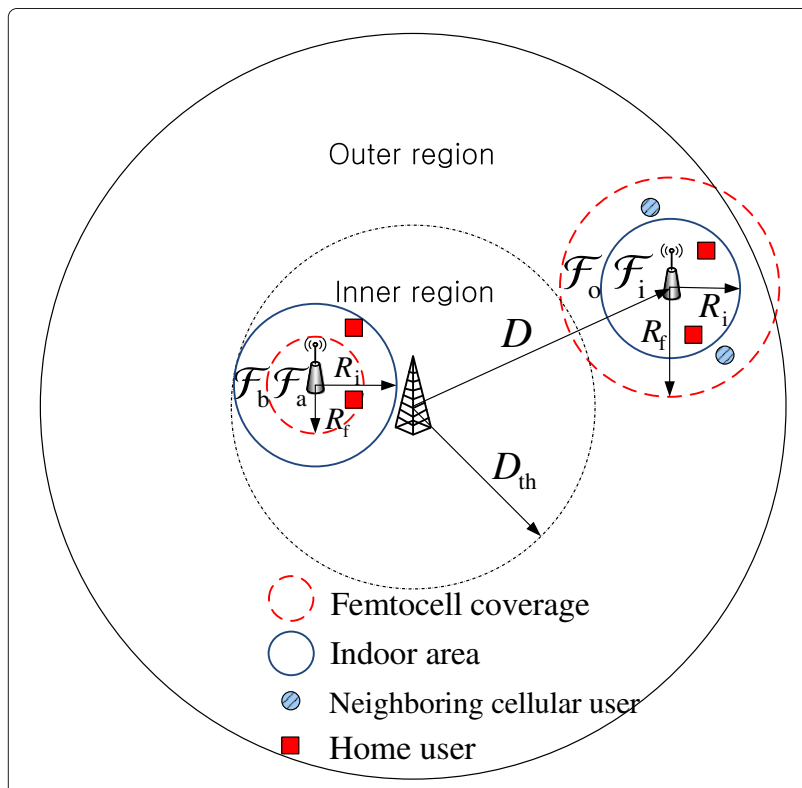

Figure 2 Femtocell coverage variation for the FAP-MBS distance $D$; in outer region $\left(D>D_{\text {th }}\right)$, femtocell coverage is larger than indoor area $\left(\boldsymbol{R}_{\mathrm{f}}>\boldsymbol{R}_{\mathrm{i}}\right)$, while $\boldsymbol{R}_{\mathrm{f}}<\boldsymbol{R}_{\mathrm{i}}$ in inner region. The femtocell coverage is classified into four geometrical zones $\mathcal{F}_{\mathrm{a}}, \mathcal{F}_{\mathrm{b}}$, $\mathcal{F}_{i}$, and $\mathcal{F}_{\text {o }}$.

in the outer region where the femtocell coverage area is larger than the indoor area, the neighboring cellular users would like to connect to the open access FAP. In conclusion, the SIR of each type of users needs a slightly different SIR model for the femtocell location (inner region or outer region) and femtocell access control (open or closed). We thus classify femtocell coverage into four geographic zones, whereby users in the same zone have the same SIR model (See Figure 2).

When an FAP is in inner region, its coverage is divided into $\mathcal{F}_{\mathrm{a}}$ and $\mathcal{F}_{\mathrm{b}}$ as follows.

- $\mathcal{F}_{\mathrm{a}}$ : indoor area where home users are served by the FAP - a disc with radius $R_{\mathrm{f}}$.

- $\mathcal{F}_{\mathrm{b}}$ : indoor area where home users are served by the MBS-a circular annulus with inner radius $R_{\mathrm{f}}$ and outer radius $R_{i}$.

When an FAP is in outer region, its coverage is divided into $\mathcal{F}_{i}$ and $\mathcal{F}_{\circ}$ as follows.

- $\mathcal{F}_{i}$ : indoor area where home users are served by the FAP-a disc with radius $R_{i}$.

- $\mathcal{F}_{\circ}$ : nearby outdoor area where neighboring cellular users are served by the FAP (in open access) or the MBS (in closed access) - a circular annulus with inner radius $R_{\mathrm{i}}$ and outer radius $R_{\mathrm{f}}$.

Although the cell coverage model based on multiple geographic zones, i.e., with multiple SIR model, is more intricate than conventional model $[9,12]$ with single SIR model for closed access only, it is essential for a conclusive comparative study of open, closed, and shared access. We do not compute the throughput of cellular users outside the four zones, since the throughput is not hardly affected by the femtocell access control, so it does not change the cellular user's preference on femtocell access.

\section{Per-zone average SIR and throughput}

Consider a reference FAP at distance $D$ from a central MBS, and its home users (or neighboring cellular users) at distance $R$ and $D_{\mathrm{C}}$ from the FAP and the MBS, respectively. As shown in Section 2.2, according to SIR model, all the users divided into four groups located in the zone $\mathcal{F}_{\mathrm{a}}$, $\mathcal{F}_{\mathrm{b}}, \mathcal{F}_{\mathrm{i}}$, and $\mathcal{F}_{\mathrm{o}}$. We analyze the throughput for the each zone, and then, based on it, derive per-tier throughput in next section. We assume small-sized femtocell $R \ll D$ resulting $D_{\mathrm{C}} \approx D$.

\subsection{Neighboring cellular user in zone $\mathcal{F}_{\text {o }}$}

Since the neighboring cellular users want to hand off to the FAP, they communicate with the FAP (open access) or MBS (closed access), which results in different SIR according to the access scheme as follows:

$$
\gamma(R)= \begin{cases}\frac{P_{\mathrm{C}} g_{0} D^{-\alpha}}{P_{\mathrm{f}} L h_{0} R^{-\alpha}+\sum_{X_{j} \in \Phi \backslash X_{0}} P_{\mathrm{f}} L h_{j}\left|X_{j}\right|^{-\alpha}} & \text { Closed Access } \\ \frac{P_{\mathrm{f}} L h_{0} R^{-\alpha}}{P_{\mathrm{C}} g_{0} D^{-\alpha}+\sum_{X_{j} \in \Phi \backslash X_{0}} P_{\mathrm{f}} L h_{j}\left|X_{j}\right|^{-\alpha}} & \text { Open Access }\end{cases}
$$

where $g_{0}$ is the exponentially distributed channel power (with unit mean) from the MBS. $h_{j}$ is the exponentially distributed channel power (with unit mean) from the FAP. $\left|X_{j}\right|$ denotes the distance between the user and the FAP. The following lemma quantifies the user SIR for the zone $\mathcal{F}_{\circ}$.

Lemma 2. The CDF of spatially averaged SIR over $\mathcal{F}_{\mathrm{O}}$, a circular annulus with outer radius $R_{\mathrm{f}}$ and inner radius $R_{\mathrm{i}}$, is given as follows

(1) Closed access:

$$
S_{\circ}^{\mathrm{CA}}(\Gamma)=1-\frac{e^{-\lambda C(K \Gamma)^{2 / \alpha}}}{R_{\mathrm{f}}^{2}-R_{\mathrm{i}}^{2}}\left(A\left(R_{f}, \alpha\right)-A\left(R_{i}, \alpha\right)\right)
$$

$$
A(x, y)=x^{2}\left(1-{ }_{2} F_{1}\left[2 / y, 1 ; 1+2 / y ;-x^{y} /(K \Gamma)\right]\right)
$$

where $C=\frac{2 \pi^{2}}{\alpha} \csc \left(\frac{2 \pi}{\alpha}\right), K=\frac{P_{\mathrm{f}} L D^{\alpha}}{P_{\mathrm{C}}}$, and ${ }_{2} F_{1}[\cdot]$ is the Gauss hypergeometric function. 
(2) Open access:

$$
S_{\circ}^{\mathrm{OA}}(\Gamma)=1-\frac{1}{R_{\mathrm{f}}^{2}-R_{\mathrm{i}}^{2}} \int_{R_{\mathrm{i}}^{2}}^{R_{\mathrm{f}}^{2}} \frac{e^{-r \lambda C \Gamma^{2 / \alpha}}}{K^{-1} \Gamma r^{\alpha / 2}+1} \mathrm{~d} r .
$$

If $\alpha=4$, the closed-form expression is given as

$$
\begin{aligned}
S_{\circ}^{\mathrm{OA}}(\Gamma)=1-\frac{B\left(R_{\mathrm{f}}^{2}\right)-B\left(R_{\mathrm{i}}^{2}\right)}{\left(R_{\mathrm{f}}^{2}-R_{\mathrm{i}}^{2}\right)}, \\
B(x)=\frac{1}{\sqrt{z}}[(-\operatorname{Re}\{E(i w)\}+\operatorname{Re}\{E(x y+i w)\}) \sin (w) \\
\quad+(\operatorname{Im}\{E(i w)\}-\operatorname{Im}\{E(x y+i w)\}) \cos (w)], \\
y=-\lambda C \sqrt{\Gamma}, z=\Gamma K^{-1}, w=y / \sqrt{z}=-\lambda C \sqrt{K},
\end{aligned}
$$

where $\operatorname{Re}\{z\}$ and $\operatorname{Im}\{z\}$ represent the real and imaginary parts of $z$, respectively, $E(z)=\int_{-\infty}^{z} \frac{e^{-t}}{t} d t$ is the Exponential integral function.

Proof. See Proof of Lemma 2 in Appendix.

3.2 Home user SIR In zone $\mathcal{F}_{\mathrm{i}}, \mathcal{F}_{\mathrm{a}}$, or $\mathcal{F}_{\mathrm{b}}$

The home user SIR for the zone $\mathcal{F}_{i}$ is given as

$$
\gamma(R)=\frac{P_{\mathrm{f}} h_{0} R^{-\beta}}{P_{\mathrm{C}} L g_{0} D^{-\alpha}+\sum_{X_{j} \in \Phi \backslash X_{0}} P_{\mathrm{f}} L^{2} h_{j}\left|X_{j}\right|^{-\alpha}},
$$

This is the same as the cellular user SIR for open access given in (4) except for the distinction that this home user is indoors and so the propagation terms are adjusted accordingly. The SIR distribution of the home user in $\mathcal{F}_{i}$ is given in the following lemma.

Lemma 3. The CDF of spatially averaged SIR over the zone $\mathcal{F}_{i}$, a disc with radius $R_{i}$, is given as

$$
S_{\mathrm{i}}(\Gamma)=1-\frac{2}{R_{\mathrm{i}}^{2}} \int_{0}^{R_{\mathrm{i}}} \frac{r \cdot e^{-\lambda C\left(L^{2} \Gamma\right)^{2 / \alpha} r^{2 \beta / \alpha}}}{K \Gamma r^{\beta}+1} d r,
$$

where $K=\frac{P_{\mathrm{C}} L}{P_{\mathrm{f}} D^{\alpha}}$. If $\alpha=4$ and $\beta=2$, then the closed-form expression is given as

$$
\begin{aligned}
& S_{\mathrm{i}}(\Gamma)= 1-H\left(R_{\mathrm{i}}\right) / R_{\mathrm{i}}^{2}, \\
& H(x)= \frac{2}{z}[(\operatorname{Re}\{E(x y+i w)\}-\operatorname{Re}\{E(i w)\}) \cos (w) \\
&+(\operatorname{Im}\{E(x y+i w)\}-\operatorname{Im}\{E(i w)\}) \sin (w)], \\
& y=-\lambda C \sqrt{\Gamma} L, z=K \Gamma, w=y / \sqrt{z}=-\lambda C L / \sqrt{K},
\end{aligned}
$$

Or, if $\alpha=\beta=4$, then

$$
S_{\text {i }}(\Gamma)=1-B\left(R_{i}^{2}\right) / R_{i}^{2},
$$

where $w, y$, and $z$ for calculating $B\left(R_{i}^{2}\right)$ are given in (12).
Proof. See Proof of Lemma 3 in Appendix.

Note that the user SIR in the zone $\mathcal{F}_{\mathrm{a}}$ is given in (9), since the zone is the indoor area covered by an FAP at $D \leq D_{\text {th }}$ (in the inner region). The SIR distribution of the home users in $\mathcal{F}_{\mathrm{a}}$ is given in the following corollary.

Corollary 1. The user SIR in the zone $\mathcal{F}_{\mathrm{a}}$ is given by (9) and $\mathcal{F}_{\mathrm{a}}$ is a disc with radius $R_{\mathrm{f}}$. Thus, the CDF of spatially averaged SIR over the zone $\mathcal{F}_{\mathrm{a}}$ is given by (10) with $R_{\mathrm{f}}$ replacing $R_{\mathrm{i}}$, i.e.,

$$
S_{\mathrm{a}}(\Gamma)=\left.S_{\text {i }}(\Gamma)\right|_{R_{i}=R_{f}} .
$$

The zone $\mathcal{F}_{\mathrm{b}}$ exists for the femtocells with $D \leq D_{\mathrm{th}}$ (in the inner region). The user SIR is

$$
\gamma(R)=\frac{P_{\mathrm{C}} g_{0} L D^{-\alpha}}{P_{\mathrm{f}} h_{0} R^{-\beta}+\sum_{X_{j} \in \Phi} P_{\mathrm{f}} L^{2} h_{j}\left|X_{j}\right|^{-\alpha}} .
$$

The SIR is the same as the cellular user SIR for closed access given in (4) except for the distinction that this home user is indoors and so the propagation terms are adjusted accordingly. Thus, the SIR distribution for $\mathcal{F}_{\mathrm{b}}$ is given in similar form to (5) as shown in the following lemma.

Lemma 4. The CDF of spatially averaged SIR over the area $\mathcal{F}_{\mathrm{b}}$, a circular annulus with outer radius $R_{\mathrm{i}}$ and inner radius $R_{\mathrm{f}}$, is given as

$$
S_{\mathrm{b}}(\Gamma)=1-\frac{e^{-\lambda C\left(L^{2} K \Gamma\right)^{2 / \alpha}}}{R_{\mathrm{i}}^{2}-R_{\mathrm{f}}^{2}}\left(A\left(R_{\mathrm{i}}, \beta\right)-A\left(R_{\mathrm{f}}, \beta\right)\right) .
$$

where $K=\frac{P_{\mp} D^{\alpha}}{P_{\mathrm{C}} L}$.

Proof. See Proof of Lemma 4 in Appendix.

Finally, combining Equation (2) and the SIR distribution given in Equations (5), (6), (10), (14), and (16), the average throughput of each zone $\mathcal{F}_{\mathrm{a}}, \mathcal{F}_{\mathrm{b}}, \mathcal{F}_{\mathrm{i}}$, and $\mathcal{F}_{\mathrm{o}}$ is given as

$$
T_{\mathrm{x}}(D)=\sum_{n=1}^{N} r_{n}\left\{S_{\mathrm{x}}\left(\Gamma_{n+1}\right)-S_{\mathrm{x}}\left(\Gamma_{n}\right)\right\}, \mathrm{x} \in\{\mathrm{a}, \mathrm{b}, \mathrm{i}, \mathrm{o}\}
$$

\subsection{Numerical results}

Figure 3 shows the spatially averaged throughput for zone $\mathcal{F}_{\mathrm{a}}, \mathcal{F}_{\mathrm{b}}, \mathcal{F}_{\mathrm{i}}$, and $\mathcal{F}_{\mathrm{o}}$ versus FAP-MBS distance for a different number of femtocells. Here, the system parameters in Table 1 are used. For the zone $\mathcal{F}_{\circ}$, we show two results $T_{\circ}^{\mathrm{CA}}$ (closed access) and $T_{\circ}^{\mathrm{OA}}$ (open access). The analytic curves given from Equation (17) are very close to the simulated curves. Furthermore, there is not a considerable difference in throughput between the inter-macrocell interference (marked with "o" and " $\square$ ") and no inter-macrocell interference (marked with " $x$ " and " + "), which validates our assumption that neglecting inter-macrocell interference 


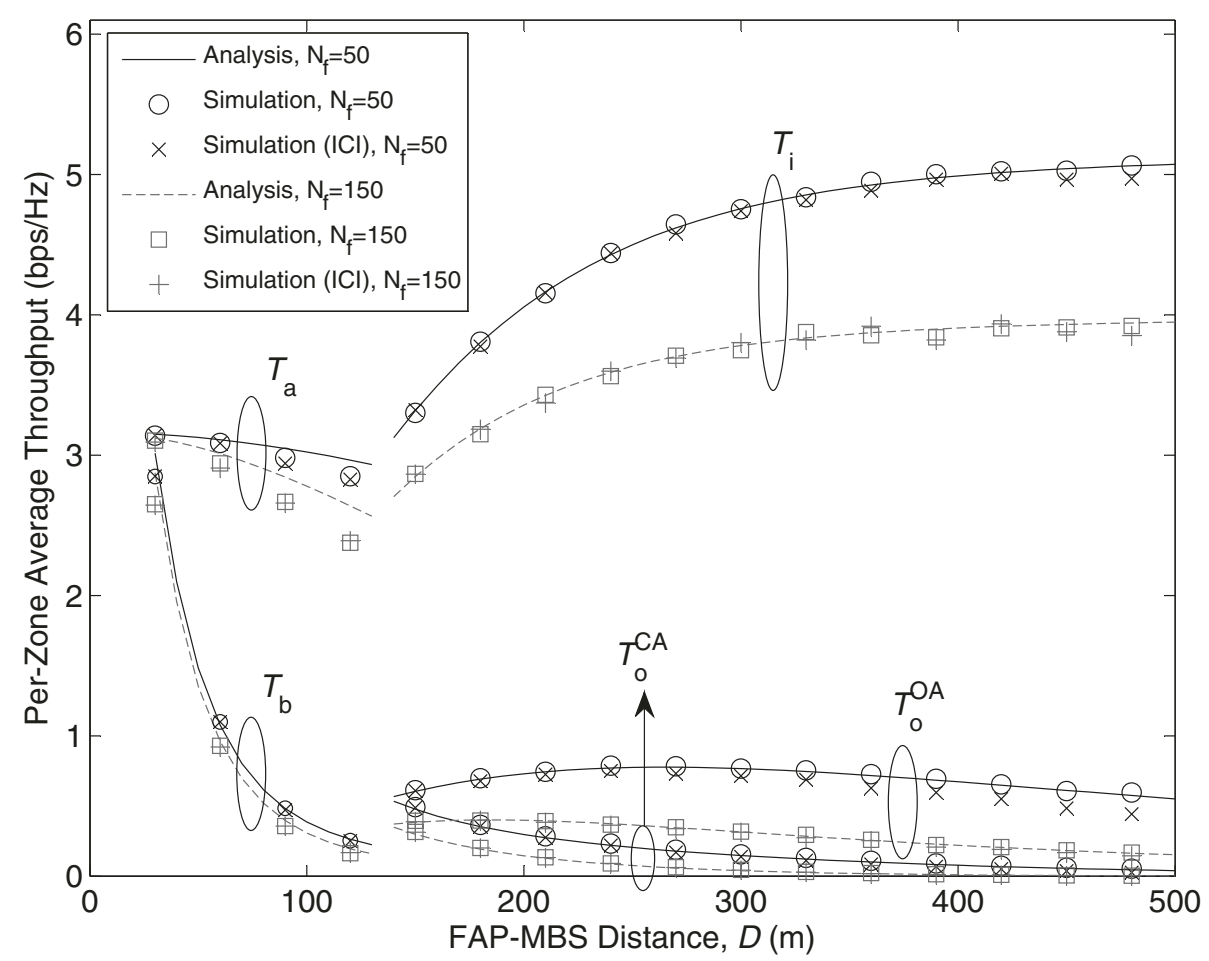

Figure 3 Theoretical and simulation results of spatially averaged throughput for the zone $\mathcal{F}_{\mathrm{a}}, \mathcal{F}_{\mathrm{b}}, \mathcal{F}_{\mathrm{i}}$, and $\mathcal{F}_{\mathrm{o}}\left(D_{\mathrm{th}}=130 \mathrm{~m}\right)$.

is acceptable in dense deploy femto. The higher interference from neighboring femtocells caused by higher $N_{\mathrm{f}}$ degrades the averaged throughput of all the zones.

\section{Per-tier throughput: closed access versus open access}

In this section, we analyze the per-tier sum throughput of closed and open accesses based on the number of users in the zone as well as the per-zone throughput obtained in preceding section. Denote $U_{\mathrm{a}}, U_{\mathrm{b}}, U_{\mathrm{i}}$, and $U_{\circ}$ are the number of users in the zone $\mathcal{F}_{\mathrm{a}}, \mathcal{F}_{\mathrm{b}}, \mathcal{F}_{\mathrm{i}}$, and $\mathcal{F}_{\mathrm{o}}$, respectively. Let $U_{\mathrm{C}}$ and $U_{\mathrm{h}}$ denote the number of outdoor cellular users and the number of home users per femtocell, respectively, and $\lambda_{C}$ and $\lambda_{h}$ are the corresponding user density. Average number of user in a macrocell cite is then given by

$$
\begin{gathered}
U=U_{\mathrm{C}}+N_{\mathrm{f}} U_{\mathrm{h}}=U_{\mathrm{C}}+\left(N_{\mathrm{f} 1}+N_{\mathrm{f} 2}\right) U_{\mathrm{h}} \\
\stackrel{(a)}{=} U_{\mathrm{C}}+N_{\mathrm{f} 1}\left(U_{\mathrm{a}}+U_{\mathrm{b}}\right)+N_{\mathrm{f} 2} U_{\mathrm{i}},
\end{gathered}
$$

where $N_{\mathrm{f} 1}=N_{\mathrm{f}}\left(D_{\mathrm{th}} / R_{\mathrm{C}}\right)^{2}$ and $N_{\mathrm{f} 2}=N_{\mathrm{f}}(1-$ $\left.\left(D_{\mathrm{th}} / R_{\mathrm{C}}\right)^{2}\right)$ are, respectively, the average number of femtocells in the inner region $\left(D \leq D_{\mathrm{th}}\right)$ and the outer region $\left(D_{\text {th }}<D \leq R_{\mathrm{C}}\right)$. Furthermore, $(a)$ follows from that $U_{\mathrm{h}}=U_{\mathrm{i}}$ in the outer region and $U_{\mathrm{h}}=U_{\mathrm{a}}+U_{\mathrm{b}}$ in the inner region.

\subsection{Closed access}

The femtocell/macrocell access scenario is different for inner and outer regions, from which the following theorem quantifies sum throughput of home user and neighboring cellular user.

Theorem 1. In closed access, the average sum throughput of home users $T_{\mathrm{h}}^{\mathrm{CA}}$ and neighboring cellular users $T_{\mathrm{C}}^{\mathrm{CA}}$ with respect to an FAP at distance D from a central MBS is given as

$$
\begin{aligned}
& T_{\mathrm{h}}^{\mathrm{CA}}(D)= \begin{cases}T_{\mathrm{a}}(D)+\rho_{\mathrm{b}}^{\mathrm{CA}} T_{\mathrm{b}}(D) & D \leq D_{\mathrm{th}} \\
T_{\mathrm{i}}(D) & D>D_{\mathrm{th}}\end{cases} \\
& T_{\mathrm{C}}^{\mathrm{CA}}(D)=\rho_{\circ} T_{\circ}^{\mathrm{CA}}(D), \quad D>D_{\mathrm{th}}
\end{aligned}
$$

where the per-zone throughput $T_{\mathrm{a}}(D), T_{\mathrm{b}}(D), T_{\mathrm{i}}(D)$, and $T_{\circ}^{\mathrm{CA}}(D)$ is given from Equation (17). $\rho_{\mathrm{b}}^{\mathrm{CA}}$ and $\rho_{\circ}$ are the fraction of time-slot dedicated to the home users in $\mathcal{F}_{\mathrm{b}}$ and the cellular users in $\mathcal{F}_{\mathrm{O}}$, respectively, among all users supported by the MBS, which is given as

$$
\begin{aligned}
\rho_{\mathrm{b}}^{\mathrm{CA}} & =\frac{R_{\mathrm{i}}^{2}-\kappa^{-2 / \alpha} D^{2}}{\frac{\lambda_{\mathrm{C}}}{\lambda_{\mathrm{h}}}\left(R_{\mathrm{C}}^{2}-N_{\mathrm{f}} R_{\mathrm{i}}^{2}\right)+\frac{1}{2} N_{\mathrm{f} 1} R_{i}^{2}}, \\
\rho_{\mathrm{o}} & =\frac{\kappa^{-2 / \alpha} D^{2}-R_{i}^{2}}{\left(R_{\mathrm{C}}^{2}-N_{\mathrm{f}} R_{\mathrm{i}}^{2}\right)+\frac{\lambda_{\mathrm{h}}}{2 \lambda_{\mathrm{c}}} N_{\mathrm{f} 1} R_{i}^{2}} .
\end{aligned}
$$

Proof. See Proof of Theorem 1 in Appendix. 
Remark 1. Interestingly, the average sum throughput of home users is lower for a farther FAP within the inner region $\left(D \leq D_{\mathrm{th}}\right)$ but higher for a farther FAP within the outer region $\left(D>D_{\mathrm{th}}\right)$. It follows that from Figure 3, increasing $D$ enhances $T_{\mathrm{i}}$ but reduces $T_{\mathrm{a}}$ and $T_{\mathrm{b}}$, and from Equation (21), increasing $D$ reduces $\rho_{\mathrm{b}}^{\mathrm{CA}}$. Intuitively, the signal from the MBS is interference to home users in the outer region, but it is the desired signal to some home users connecting to the MBS in the inner region.

Remark 2. The per-zone throughput $T_{\mathrm{i}}, T_{\mathrm{a}}$, and $T_{\mathrm{b}}$ are not affected by $\lambda_{\mathrm{C}}$. From Equation (21), increasing $\lambda_{\mathrm{C}}$ reduces $\rho_{\mathrm{b}}^{\mathrm{CA}}$. Intuitively, this means that an MBS load is higher at more cellular user deployment and thereby fewer time slots are allocated home user served by the MBS. For an FAP in inner region, $T_{h}^{C A}$ is thus higher for a lower cellular user density, while it is independent of cellular user density in outer region. Since from Equation (22) $\rho_{\circ}$ is higher at a larger $U_{\mathrm{C}}$, but $T_{\circ}^{\mathrm{CA}}$ is independent on $U_{\mathrm{C}}$, higher cellular user density increases the average sum throughput of neighboring cellular user.

\subsection{Open access}

Theorem 2. In open access, the average sum throughput of home users, $T_{\mathrm{h}}^{\mathrm{OA}}$, and neighboring cellular users, $T_{\mathrm{C}}^{\mathrm{OA}}$, with respect to a FAP at distance $D$ from a central $M B S$ is given as

$$
\begin{aligned}
& T_{\mathrm{h}}^{\mathrm{OA}}(D)= \begin{cases}T_{\mathrm{a}}(D)+\rho_{\mathrm{b}}^{\mathrm{OA}} T_{\mathrm{b}}(D) & D \leq D_{\mathrm{th}} \\
\rho_{\mathrm{i}} T_{\mathrm{i}}(D) & D>D_{\mathrm{th}}\end{cases} \\
& T_{\mathrm{C}}^{\mathrm{OA}}(D)=\left(1-\rho_{\mathrm{i}}\right) T_{\mathrm{OA}}^{\mathrm{OA}}(D), \quad D>D_{\mathrm{th}}
\end{aligned}
$$

where $T_{\circ}^{\mathrm{OA}}(D)$ is given from Equation (17). $\rho_{\mathrm{b}}^{\mathrm{OA}}$ is the fraction of time-slot dedicated to the home users in $\mathcal{F}_{\mathrm{b}}$ among home and cellular users supported from the $M B S$, and $\rho_{i}$ is the fraction of time-slot dedicated to the home users in $\mathcal{F}_{i}$ among home and cellular users supported from the FAP. They are given as

$$
\begin{aligned}
\rho_{\mathrm{b}}^{\mathrm{OA}} & =\frac{R_{\mathrm{i}}^{2}-\kappa^{-2 / \alpha} D^{2}}{\frac{\lambda_{\mathrm{c}}}{\lambda_{\mathrm{h}}}\left(R_{\mathrm{C}}^{2}-N_{\mathrm{f} 1} R_{\mathrm{i}}^{2}-\frac{\kappa^{-2 / \alpha}}{2} N_{\mathrm{f} 2}\left(R_{\mathrm{C}}^{2}+D_{\mathrm{th}}\right)\right)+\frac{1}{2} N_{\mathrm{f} 1} R_{i}^{2}} \\
\rho_{\mathrm{i}} & =\left(1+\frac{\lambda_{\mathrm{C}}}{\lambda_{\mathrm{h}}}\left(\frac{\kappa^{-2 / \alpha} D^{2}}{R_{\mathrm{i}}^{2}}-1\right)\right)^{-1}
\end{aligned}
$$

Proof. See Proof of Theorem 2 in Appendix.

Remark 3. In the inner region, $T_{\mathrm{h}}^{\mathrm{OA}}$ is lower at farther FAP, since increasing $D$ reduces $\rho_{\mathrm{b}}^{\mathrm{OA}}$ in Equation (25) as well as $T_{\mathrm{a}}$ and $T_{\mathrm{b}}$ in Figure 3 . Whereas, in the outer region, as $D$ increases, $T_{\mathrm{h}}^{\mathrm{OA}}$ increase and begins to decrease at sufficiently large $D$. This is because $T_{i}$ is upper limited by the highest order modulation in spite of quite high SIR at large $D$, while $\rho_{i}$ decreases to zero. Next, from Equation (26), $1-\rho_{i}$ is higher for larger $\lambda_{C}$ or $D$, which enhances $T_{\mathrm{C}}^{\mathrm{OA}}$.

The throughput comparison of both the access schemes is given in the remarks below.

Remark 4. First, $T_{\mathrm{h}}^{\mathrm{CA}} \leq T_{\mathrm{h}}^{\mathrm{OA}}$ for the inner region, because closed access rather than open access increases the number of users supported by the MBS and thereby $\rho_{\mathrm{b}}^{\mathrm{CA}} \leq \rho_{\mathrm{b}}^{\mathrm{OA}}$. Intuitively, open access femtocells in the outer region admit neighboring cellular users which reduces the macrocell load. This effectively increases the throughput of some home users (supported by the MBS) in inner region. Whereas, $T_{\mathrm{h}}^{\mathrm{CA}} \geq T_{\mathrm{h}}^{\mathrm{OA}}$ for the outer region because $\rho_{\mathrm{i}} \leq 1$ from Equation (26). Second, we observe $T_{\mathrm{C}}^{\mathrm{CA}}<$ $T_{\mathrm{C}}^{\mathrm{OA}}$. Since $U_{\mathrm{i}}+U_{\circ}<U_{\mathrm{C}}+N_{\mathrm{f} 1} \bar{U}_{\mathrm{b}}$, comparing $1-\rho_{\mathrm{i}}=$ $\frac{U_{\circ}}{U_{i}+U_{\circ}}$ and Equation (53) yields $1-\rho_{i}>\rho_{\circ}$. Moreover, $T_{\circ}^{\mathrm{OA}}$ is obviously larger than $T_{\circ}^{\mathrm{CA}}$. In conclusion, home user and neighboring cellular user prefer opposite access schemes.

\subsection{Numerical results}

The throughput results in this section are obtained with the system parameters in Table 1 . Figures 4 and 5 show the home user throughput analytically obtained using (19) and (23) versus FAP-MBS distance $D$ for different numbers of femtocells $N_{\mathrm{f}}$ and cellular users $U_{\mathrm{C}}$. Since $R_{\mathrm{f}}=R_{\mathrm{i}}$ at $D=D_{\text {th }}$, we obtain the distance $D_{\text {th }}$ of $130 \mathrm{~m}$ by substituting $R_{i}=20$ into Equation (3). In closed access, home user throughput decreases with $D\left(D \leq D_{\text {th }}\right)$, while it increases with $D\left(D>D_{\text {th }}\right)$ per Remark 1. For $D>D_{\text {th }}$, the home user throughput in open access first increases then decreases with increasing $D$. Additionally, Figure 5 shows that the turning point moves into the cell interior with increasing $U_{\mathrm{C}}$. This is because increasing $D$ and $U_{\mathrm{C}}$ increases the number of neighboring cellular users, and thus, the time resource allocated to home user in femtocell downlink is reduced. In Figure 4, the throughput for both open and closed accesses is degraded, since the aggregated interference from other femtocells increases with $N_{\mathrm{f}}$. We observe that unlike the case of $D>D_{\mathrm{th}}$, open access outperforms closed access for $D \leq D_{\text {th }}$. However, the throughput loss of open access at $D>D_{\text {th }}$ dominates the throughput gain at $D \leq D_{\text {th }}$. Thus, closed access is better for home users.

Figure 6 plots the sum throughput of neighboring cellular users of a reference femtocell using Equations (20) and (24). The throughput is high at a low femtocell density and a high cellular user density, which agrees with the prediction in Remarks 2 and 3. The throughput $\left(T_{\mathrm{C}}^{\mathrm{CA}}<\right.$ $0.003 \mathrm{bps} / \mathrm{Hz}$ ) in closed access is too low to offer typical services $(0.003 \mathrm{bps} / \mathrm{Hz}$ is equivalent to $15 \mathrm{kbps}$ for 


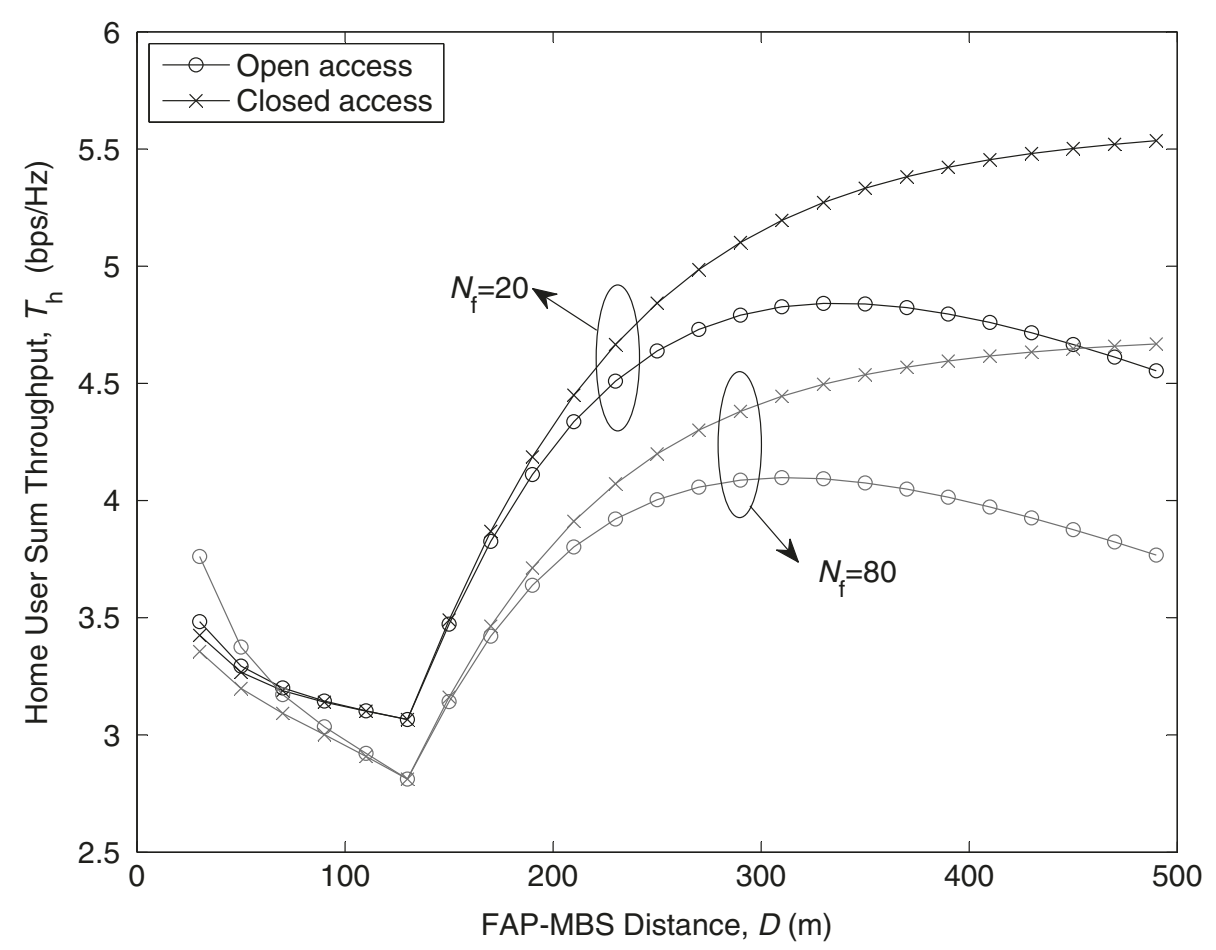

Figure 4 Average sum throughput of the home users for different number of femtocells $\left(D_{\mathrm{th}}=130 \mathrm{~m}\right.$, number of cellular users $\left.U_{\mathrm{c}}=20\right)$.

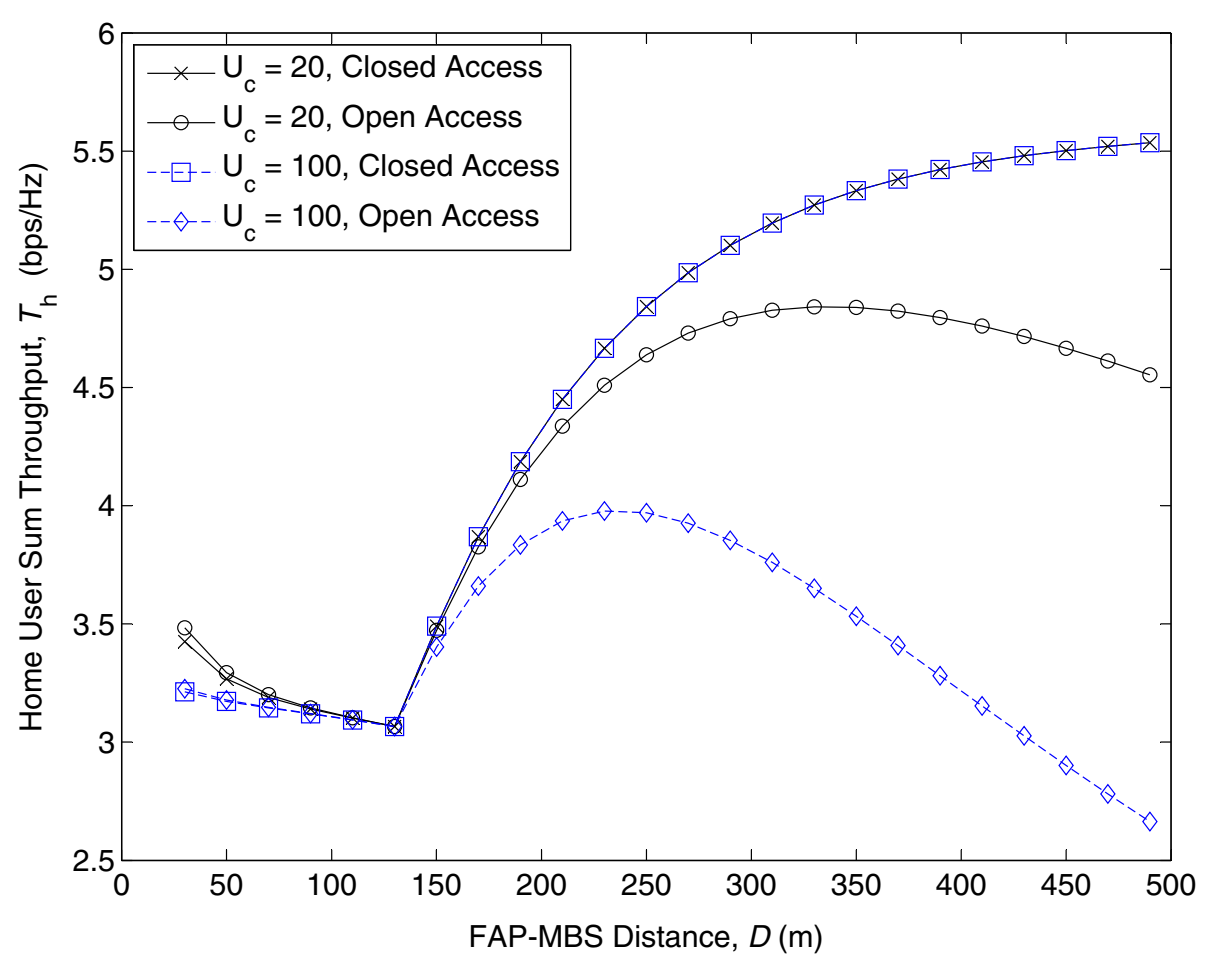

Figure 5 Average sum throughput of the home users for different number of cellular users $\left(D_{\text {th }}=130 \mathrm{~m}\right.$, number of femtocells $\left.N_{\mathrm{f}}=20\right)$. 


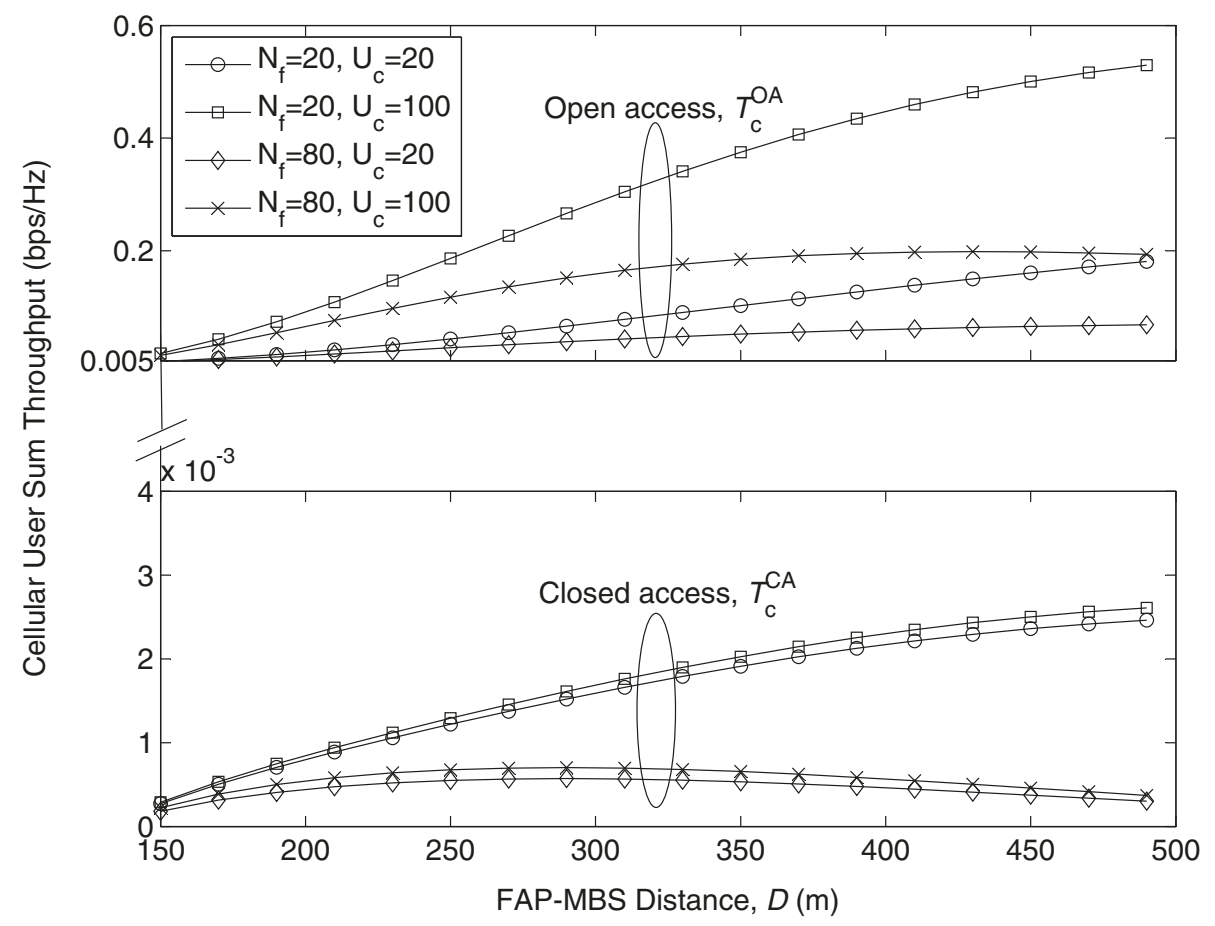

Figure 6 Average sum throughput of the neighboring cellular users for different number of femtocells and cellular users $\left(D_{\mathrm{th}}=130 \mathrm{~m}\right)$.

5-MHz bandwidth). Thus, open access is much better for neighboring cellular users, in contrast to the result for home users. Table 2 summarizes these results of closed versus open access.

Figure 7 plots the network throughput in open access and closed access, sum of home user and neighboring cellular user throughput, i.e., $T^{\mathrm{CA}}=T_{\mathrm{h}}^{\mathrm{CA}}+T_{\mathrm{C}}^{\mathrm{CA}}=T_{\mathrm{i}}+$ $\rho_{\circ} T_{\circ}^{\mathrm{CA}}$ and $T^{\mathrm{OA}}=T_{\mathrm{h}}^{\mathrm{OA}}+T_{\mathrm{C}}^{\mathrm{OA}}=\rho_{\mathrm{i}} T_{\mathrm{i}}+\left(1-\rho_{\mathrm{i}}\right) T_{\circ}^{\mathrm{OA}}$. Note that for $D>D_{\mathrm{th}}$, open access is inferior to closed access with respect to the network throughput. The reason is from the inequality given by $T^{\mathrm{CA}}-T^{\mathrm{OA}}=\left(1-\rho_{\mathrm{i}}\right)\left(T_{\mathrm{i}}-\right.$ $\left.T_{\circ}^{\mathrm{OA}}\right)+\rho_{\circ} T_{\circ}^{\mathrm{CA}}>0$. Intuitively, since $T_{\mathrm{i}}-T_{\circ}^{\mathrm{OA}}>T_{\circ}^{\mathrm{OA}}-T_{\circ}^{\mathrm{CA}}$ from Figure 3, the decrement of home user throughput $T_{\mathrm{i}}$ due to time resource sharing with cellular users in

Table 2 Throughput comparison of open, closed, and shared access for FAP-MBS distance $D$, cellular user density $U_{c}$, femtocell density $N_{\mathrm{f}}$

\begin{tabular}{|c|c|c|c|}
\hline & & $\begin{array}{l}\text { High } D \\
\text { and/or low } N_{f} \\
\text { and/or high } U_{c}\end{array}$ & $\begin{array}{l}\text { Low } D \\
\text { and/or high } N_{\mathrm{f}} \\
\text { and/or low } U_{\mathrm{c}}\end{array}$ \\
\hline \multirow[t]{4}{*}{ Home user throughput } & Inner & Open $=$ Shared $>$ Closed & Open $=$ Shared $\gg$ Closed \\
\hline & region & & \\
\hline & Outer & Closed $\gg$ Shared $\gg$ Open & Closed $>$ Shared $>$ Open \\
\hline & region & & \\
\hline \multirow{4}{*}{$\begin{array}{l}\text { Cellular user } \\
\text { throughput }\end{array}$} & & Open $\gg$ Shared $\gg$ Closed & Open $>$ Shared $>$ Closed \\
\hline & & & \\
\hline & Home & Closed access & Closed access \\
\hline & users & & \\
\hline \multirow{4}{*}{$\begin{array}{l}\text { Preferred } \\
\text { access }\end{array}$} & Cellular & Open access & Open access \\
\hline & users & & \\
\hline & Both & Shared access & Shared access \\
\hline & users & & \\
\hline
\end{tabular}




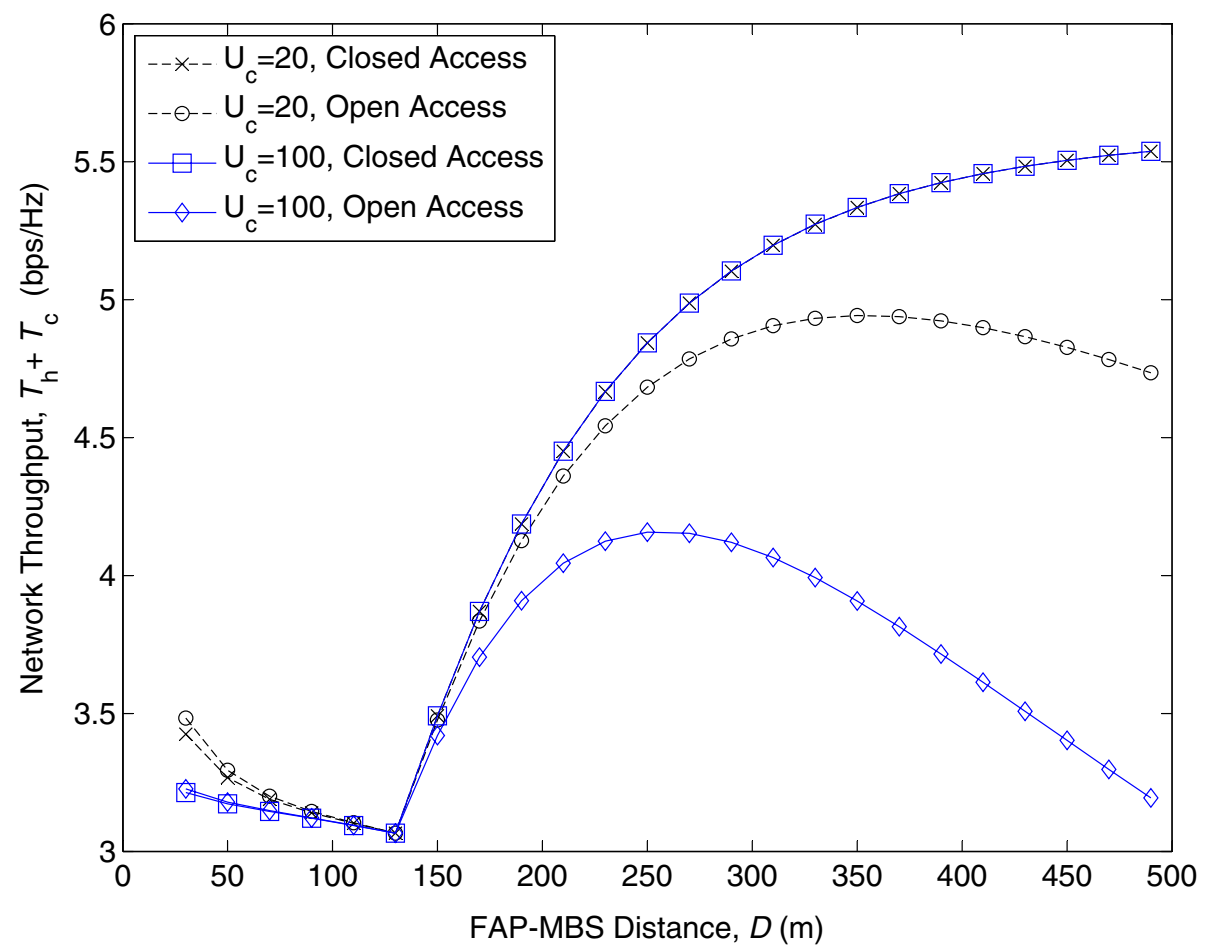

Figure 7 Network throughput for different number of cellular users, $\left(\boldsymbol{N}_{\mathrm{f}}=\mathbf{2 0}\right.$ and $\left.\boldsymbol{D}_{\mathrm{th}}=\mathbf{1 3 0 \mathrm { m }}\right)$. The two curves of closed access are the same for $D>D_{\text {th }}$.

open access prevails against the increment of cellular user throughput by substituting $T_{\circ}^{\mathrm{OA}}$ for $T_{\circ}^{\mathrm{CA}}$. In a different point of view, this implies that a slight increase in the time fraction $\rho_{\mathrm{i}}$ provides a high increase $T_{\mathrm{h}}^{\mathrm{OA}}$ at the cost of a slight drop in $T_{\mathrm{C}}^{\mathrm{OA}}$, i.e., an increase in network throughput $T^{\mathrm{OA}}$. This, as well as the extremely low throughput in closed access motivates the shard access femtocells using time slot allocation, which will be discussed in the next section.

\section{Shared access: time-slot allocation}

We consider the shared access where an FAP allocates $\eta$ fraction of time-slots to home users and the remaining $1-$ $\eta$ fraction of time-slots to cellular users. Unlike the timeslot allocation in open access, where the time fraction $\rho_{\text {i }}$ is dependent on the number of home users and cellular users, the time-slot allocation in the shared access optimizes $\eta$ to maximize the network throughput $T^{\mathrm{SA}}$ while satisfying QoS requirement. The network throughput is given as

$$
T^{\mathrm{SA}}=\eta T_{\mathrm{i}}+(1-\eta) T_{\circ}^{\mathrm{OA}}, \quad \eta \in[0,1] .
$$

We define the QoS requirement as the average user throughput $\bar{T}_{\mathrm{h}}$ (home user) and $\bar{T}_{\mathrm{C}}$ (cellular user) is larger than the required minimum throughput $\Omega_{\mathrm{h}}$ (home user) and $\Omega_{\mathrm{C}}=\varepsilon \Omega_{\mathrm{h}}$ with $\varepsilon \in(0,1]$ (cellular user), respectively.
Satisfying the QoS, the time-slot allocation problem to maximize the network throughput $T^{\mathrm{SA}}$ is formulated as

$$
\begin{aligned}
\max _{0 \leq \eta \leq 1} \eta T_{\mathrm{i}}+(1-\eta) T_{\circ}^{\mathrm{OA}} \text { subject to } \bar{T}_{\mathrm{h}} & \geq \Omega_{\mathrm{h}}, \\
\bar{T}_{\mathrm{C}} & \geq \Omega_{\mathrm{C}}=\varepsilon \Omega_{\mathrm{h}}
\end{aligned}
$$

where $\bar{T}_{\mathrm{h}}=\frac{\eta T_{\mathrm{i}}}{U_{\mathrm{i}}}$ and $\bar{T}_{\mathrm{C}}=\frac{(1-\eta) T_{\circ}^{\mathrm{OA}}}{U_{\circ}}$.

Theorem 3. The optimal value $\eta^{*}$ of the time-slot allocation in Equation (28) is given as

$$
\eta^{*}=1-\varepsilon \frac{\Omega_{\mathrm{h}} U_{\circ}}{T_{\circ}^{\mathrm{OA}}} .
$$

The solution $\eta^{*}$ is feasible when it is equal or larger than $\frac{\Omega_{\mathrm{h}} U_{\mathrm{i}}}{T_{\mathrm{i}}}$.

Proof. Denote $\mathcal{Q}_{1}$ and $\mathcal{Q}_{2}$ as a set of $\eta$ satisfying the QoS requirement (28) in the order of description, respectively. we then obtain intersection of the three sets as $\mathcal{Q}=\mathcal{Q}_{1} \cap \mathcal{Q}_{2}=\left\{\eta \mid \frac{\Omega_{\mathrm{h}} U_{\mathrm{i}}}{T_{\mathrm{i}}} \leq \eta \leq 1-\varepsilon \frac{\Omega_{\mathrm{h}} U_{\mathrm{o}}}{T_{\mathrm{o}}^{\mathrm{OA}}}\right\}$ for $\frac{\Omega_{\mathrm{h}} U_{\mathrm{i}}}{T_{\mathrm{i}}} \leq$ $1-\varepsilon \frac{\Omega_{\mathrm{h}} U_{\mathrm{o}}}{T_{\mathrm{o}}^{\mathrm{OA}}}$. Define a function of $\eta$ as $f(\eta)=\eta T_{\mathrm{i}}+(1-$

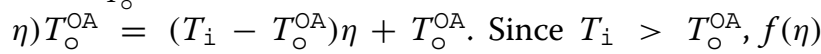
monotonically increases with increasing $\eta$. Thus, $\eta^{*}$ is the 
maximum $\eta \in \mathcal{Q}$, which yields Equation (29). Moreover, since $\mathcal{Q}=\varnothing$ for $\frac{\Omega_{\mathrm{h}} U_{\mathrm{i}}}{T_{\mathrm{i}}}>1-\varepsilon \frac{\Omega_{\mathrm{h}} U_{\mathrm{o}}}{T_{\mathrm{O}}^{\mathrm{OA}}}, \eta^{*}$ is feasible for $\eta^{*} \geq \frac{\Omega_{\mathrm{h}} U_{\mathrm{i}}}{T_{\mathrm{i}}}$.

Remark 5. Shared access with $\eta^{*}=1$ and $\eta^{*}=\rho_{i}=$ $\frac{U_{i}}{U_{i}+U_{o}}$ is closed and open accesses, respectively. The QoS parameter $\varepsilon \in(0,1]$ determines the priority of home users relative to cellular users with $\varepsilon=1$ ensuring identical required minimum throughput to home and cellular users. In Equation (29), increasing $\varepsilon$ reduces $\eta^{*}$ and allocates more time-slots to cellular users. This indicates that shared access provides lower network throughput than open access when $\varepsilon$ is set high, e.g., such that $\eta^{*}<\frac{U_{i}}{U_{i}+U_{o}}$.

Figure 8 compares the network throughput for different femtocell access schemes, where the system parameters in Table 1 are adopted. We assume the QoS requirement $\Omega_{\mathrm{h}}=0.01$ (corresponding to $500 \mathrm{kbps}$ for $5-\mathrm{MHz}$ bandwidth) and $\Omega_{\mathrm{c}}=0.01 \varepsilon$. Since $R_{\mathrm{f}}=R_{\mathrm{i}}$ at $D=D_{\mathrm{th}}$, we obtain the distance $D_{\text {th }}$ of $130 \mathrm{~m}$ by substituting $R_{i}=20$ into Equation (3). For $D>D_{\mathrm{th}}$, the throughput of shared access increases with decreasing $\varepsilon$. Considering lower $\varepsilon$ results in higher $\eta$, this indicates that increasing home user throughput $\eta T_{\mathrm{i}}$ counteracts the effects of decreasing cellular user throughput $(1-\eta) T_{\circ}^{\mathrm{OA}}$. Moreover, this implies that the shared access with higher $\varepsilon$ (more timeslot allocation to cellular users) provides lower throughput than open access as shown in the result for $\varepsilon=0.1$. For $D>D_{\text {th }}$, closed access always provides higher throughput than shared access because shared access with $\eta=1$, which does not satisfy the QoS requirement, is the same as closed access. For $D \leq D_{\mathrm{th}}$, shared access obtains the same throughput as open access regardless of $\varepsilon$. The reason is that like open access, the shared access with time-slot allocation allows access from all neighboring cellular users located in the zone $\mathcal{F}_{\mathrm{O}}$. Note that the shared access with appropriate value of $\varepsilon$ achieves higher (at $D>D_{\text {th }}$ ) or equal (at $D \leq D_{\text {th }}$ ) network throughput than open access. We summarize these observations in Table 2 .

\section{Conclusion}

The overall contribution of this article is a new analytical framework for evaluating throughput tradeoffs regarding femtocell access schemes in downlink two-tier femtocell networks. The framework quantifies femtocellsite-specific "loud neighbor" effects and can be used to compare other techniques, e.g., power control, spectrum allocation, and MIMO. Our results show that unlike the uplink results in [17], the preferred access scheme for home and cellular users is incompatible. In particular, closed access provides higher throughput for home users and lower throughput for neighboring cellular users; vice versa with open access. As a compromise, we suggest shared access where femtocells choose a time-slot ratio for their home and neighboring cellular users to maximize the network throughput subject to a network-wide QoS requirement. For femtocells within the outer area, shared

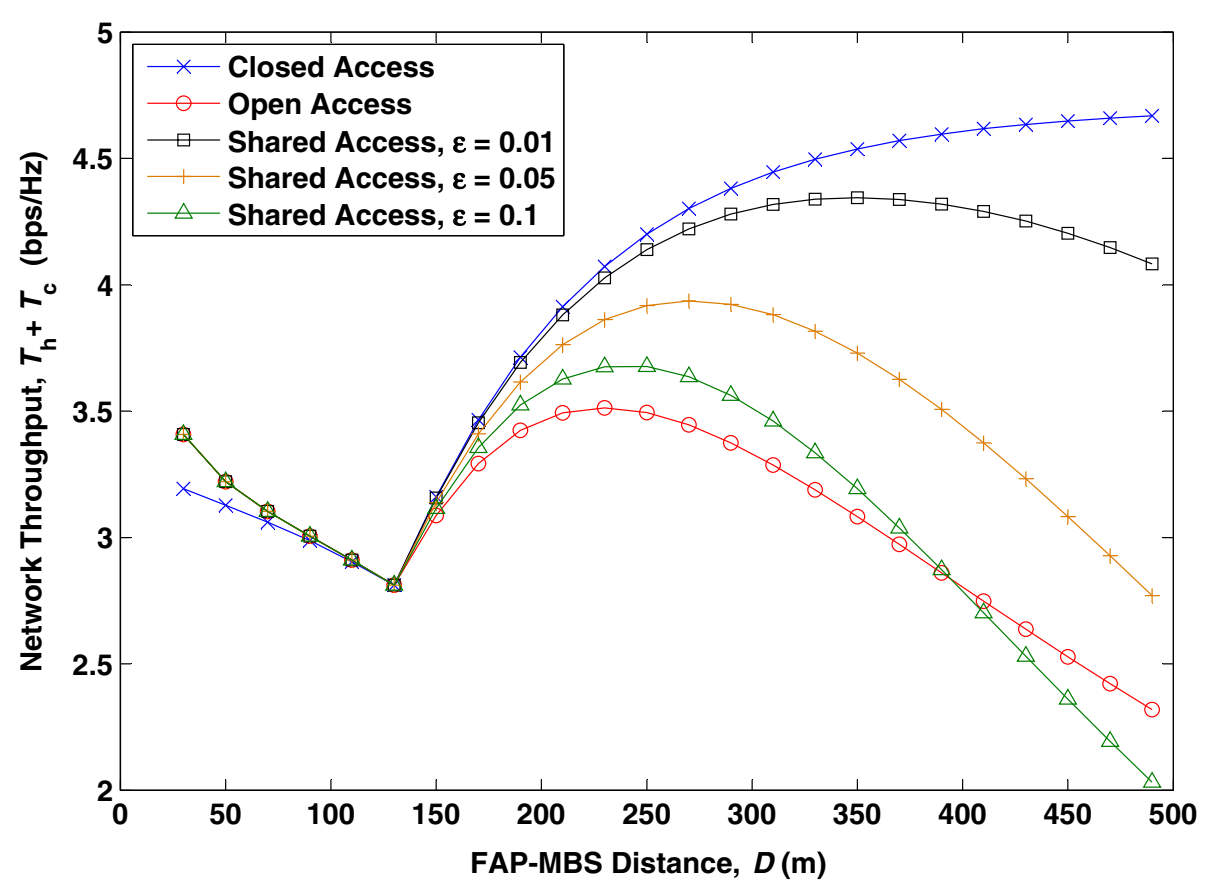

Figure 8 Network throughput for different femtocell access, $\left(N_{\mathrm{f}}=80, U_{\mathrm{c}}=100\right.$, and $\left.D_{\mathrm{th}}=130 \mathrm{~m}\right)$. 
access achieves higher network throughput than open access while satisfying the QoS of both home and cellular users. These results motivate shared access-i.e., open access, but with limits-in femtocell-enhanced cellular networks with universal frequency reuse.

\section{Appendix}

\section{Proof of Lemma 2}

In closed access, the user SIR in Equation (4) is rewritten as $\gamma(R)=\frac{g_{0}}{K\left(I_{1}+I_{2}\right)}$, where $K=\frac{P_{\mp} L}{P_{\mathrm{c}} D^{-\alpha}}$ and $I_{1}=h_{0} R^{-\alpha}$ and $I_{2}=\sum_{j \in \Phi \backslash A_{0}} h_{j}\left|X_{j}\right|^{-\alpha}$. Then, the complementary cumulative distribution function (CCDF) of the user SIR at distance $R$ from the FAP is given as

$$
\begin{aligned}
\mathbb{P}[\gamma(R) \geq \Gamma] & =\mathbb{P}\left[g_{0} \geq \Gamma K\left(I_{1}+I_{2}\right)\right] \\
& \stackrel{(a)}{=} \int_{0}^{\infty} e^{-\Gamma K s} \mathrm{~d} \mathbb{P}\left(I_{1}+I_{2} \leq s\right) \\
& =\mathcal{L}_{I_{1}}(\Gamma K) \mathcal{L}_{I_{2}}(\Gamma K),
\end{aligned}
$$

where (a) follows because the CCDF of exponential $g_{0}$ with unit mean is given as $\mathbb{P}\left[g_{0}>t\right]=e^{-t}$. Here, $\mathcal{L}_{I_{1}}(\Gamma K)$ is the Laplace transform of $I_{1}$ (exponential random variable scaled by $R^{-\alpha}$ ), which is given as

$$
\begin{aligned}
\mathcal{L}_{I_{1}}(\Gamma K) & =\int_{0}^{\infty} e^{-\Gamma K s} f_{I_{1}}(s) \mathrm{d} s=R^{\alpha} \int_{0}^{\infty} e^{-\Gamma K s} e^{-s R^{\alpha}} \mathrm{d} s \\
& =\frac{1}{\Gamma K R^{-\alpha}+1},
\end{aligned}
$$

Moreover, $\mathcal{L}_{I_{2}}(\Gamma K)$ is the Laplace transform of the Poisson shot-noise process $I_{2}$. For exponential $h_{j}$ with unit mean, $\mathcal{L}_{I_{2}}(\Gamma K)$ is given by [31]

$$
\mathcal{L}_{I_{2}}(\Gamma K)=e^{-\lambda C(K \Gamma)^{2 / \alpha}},
$$

where $C=\frac{2 \pi^{2}}{\alpha} \csc \left(\frac{2 \pi}{\alpha}\right)$. Thus, Equation (30) is simplifies to

$$
\mathbb{P}[\gamma(R) \geq \Gamma]=\frac{e^{-\lambda C(K \Gamma)^{2 / \alpha}}}{\Gamma K R^{-\alpha}+1}
$$

The cellular users are uniformly located at the zone $\mathcal{F}_{\circ}$ that a circular annulus with outer radius $R_{\mathrm{f}}$ and inner radius $R_{i}$. Then, probability density function (PDF) of the distance $R$ is $f_{R}(r)=\frac{2 r}{R_{\mathrm{f}}^{2}-R_{\mathrm{i}}^{2}}, R \in\left[R_{\mathrm{i}}, R_{\mathrm{f}}\right]$. The spatially averaged SIR distribution over $\mathcal{F}_{\circ}$ is given as

$$
\begin{aligned}
S_{\circ}^{\mathrm{CA}}(\Gamma) & =\mathbb{E}_{R}[\mathbb{P}[\gamma(R) \leq \Gamma]] \\
& =1-\frac{2 e^{-\lambda C(K \Gamma)^{2 / \alpha}}}{R_{\mathrm{f}}^{2}-R_{\mathrm{i}}^{2}} \int_{R_{\mathrm{i}}}^{R_{\mathrm{f}}} \frac{R}{\Gamma K R^{-\alpha}+1} \mathrm{~d} R .
\end{aligned}
$$

Desired result in Equation (5) is obtained by further calculating Equation (34) with the following integration formula [32]

$$
\int \frac{t}{a t^{-\alpha}+1} \mathrm{~d} t=\frac{1}{2} t^{2}\left(1-{ }_{2} F_{1}\left[\frac{2}{\alpha}, 1 ; 1+\frac{2}{\alpha} ;-\frac{t^{\alpha}}{a}\right]\right) .
$$

Next, in open access, the user SIR in (4) is rewritten as $\gamma(R)=\frac{h_{0}}{R^{\alpha}\left(I_{3}+I_{4}\right)}$, where $I_{3}=K^{-1} g_{0}$ and $I_{4}=$ $\sum_{j \in \Phi \backslash A_{0}} h_{j}\left|X_{j}\right|^{-\alpha}$. Using the same approach as in Equation (30), CCDF of the user SIR at distance $R$ from the FAP is given as

$$
\mathbb{P}[\gamma(R) \geq \Gamma]=\mathcal{L}_{I_{3}}\left(\Gamma R^{\alpha}\right) \mathcal{L}_{I_{4}}\left(\Gamma R^{\alpha}\right)=\frac{e^{-\lambda C \Gamma^{2 / \alpha} R^{2}}}{\Gamma K^{-1} R^{\alpha}+1} .
$$

From Equations (34) and (36), the spatially averaged SIR distribution over $\mathcal{F}_{\circ}$ is given as

$$
S_{\circ}^{\mathrm{OA}}(\Gamma)=1-\frac{1}{R_{\mathrm{f}}^{2}-R_{\mathrm{i}}^{2}} \int_{R_{\mathrm{i}}}^{R_{\mathrm{f}}} \frac{2 R}{\Gamma K^{-1} R^{\alpha}+1} e^{-\lambda C \Gamma^{2 / \alpha} R^{2}} \mathrm{~d} R .
$$

Here, by using substitution $R^{2}=r$, we obtain the desired result in Equation (6).

In particular, for $\alpha=4$ and by using substitution $R^{2}=r$, Equation (37) is rewritten as

$$
\begin{aligned}
S_{\circ}^{\mathrm{OA}}(\Gamma) & =1-\frac{1}{R_{\mathrm{f}}^{2}-R_{\mathrm{i}}^{2}} \int_{R_{i}^{2}}^{R_{\mathrm{f}}^{2}} \frac{e^{-r \lambda C \sqrt{\Gamma}}}{\Gamma K^{-1} r^{2}+1} \mathrm{~d} r \\
& =1-\frac{B\left(R_{\mathrm{f}}^{2}\right)-B\left(R_{\mathrm{i}}^{2}\right)}{R_{\mathrm{f}}^{2}-R_{\mathrm{i}}^{2}}
\end{aligned}
$$

with

$$
\begin{aligned}
B(x)= & \int_{0}^{x} \frac{e^{y t}}{z t^{2}+1} \mathrm{~d} t \stackrel{(a)}{=}-\frac{i}{2 \sqrt{z}}\left[e^{i y / \sqrt{z}}\{E(x y-i y / \sqrt{z})\right. \\
& -E(-i y / \sqrt{z})\}+e^{-i y / \sqrt{z}}\{E(i y / \sqrt{z}) \\
& -E(x y+i y / \sqrt{z})\}] \\
\stackrel{(b)}{=} & \frac{1}{\sqrt{z}}[(-\operatorname{Re}\{E(i y / \sqrt{z})\}+\operatorname{Re}\{E(x y+i y / \sqrt{z})\}) \\
& \times \sin (y / \sqrt{z})+(\operatorname{Im}\{E(i y / \sqrt{z})\} \\
& -\operatorname{Im}\{E(x y+i y / \sqrt{z})\}) \cos (y / \sqrt{z})],
\end{aligned}
$$

where $y=-\lambda C \sqrt{\Gamma}, z=\Gamma K^{-1}$. (a) follows from the integral formula in [33] and (b) is given on the mirror symmetry of Exponential integral function, i.e., $E(\bar{z})=$ $\overline{E(z)}$. Combining Equations (38) and (39) gives the desired result (7).

\section{Proof of Lemma 3}

The home user SIR in Equation (9) is rewritten as $\gamma(R)=$ $\frac{h_{0}}{R^{\beta}\left(I_{1}+I_{2}\right)}$, where $I_{1}=K g_{0}$ with $K=\frac{P_{\mathrm{c}} L}{P_{\mp} D^{\alpha}}$ and $I_{2}=$ $L^{2} \sum_{j \in \Phi \backslash A_{0}} h_{j}\left|X_{j}\right|^{-\alpha}$. Using the way to obtain Equation (36), 
CCDF of the user SIR at distance $R$ from the FAP is given as

$$
\begin{aligned}
\mathbb{P}[\gamma(R) \geq \Gamma] & =\mathcal{L}_{I_{1}}\left(\Gamma R^{\beta}\right) \mathcal{L}_{I_{2}}\left(\Gamma R^{\beta}\right) \\
& =\frac{e^{-\lambda C\left(L^{2} \Gamma\right)^{2 / \alpha} R^{2 \beta / \alpha}}}{\Gamma K R^{-\beta}+1},
\end{aligned}
$$

Assuming that the home users are uniformly located in $\mathcal{F}_{i}$, PDF of $R$ is $f_{R}(r)=\frac{2 r}{R_{i}^{2}}, R \in\left(0, R_{i}\right]$. Thus, the spatially averaged SIR distribution of the home users is given as

$$
\begin{aligned}
S_{\mathrm{i}}(\Gamma) & =\mathbb{E}_{R}[\mathbb{P}[\gamma(R) \leq \Gamma]] \\
& =1-\frac{2}{R_{\mathrm{i}}^{2}} \int_{0}^{R_{\mathrm{i}}} \frac{R \cdot e^{-\lambda C\left(L^{2} \Gamma\right)^{2 / \alpha} R^{2 \beta / \alpha}}}{\Gamma K R^{\beta}+1} \mathrm{~d} R,
\end{aligned}
$$

which proves Equation (10).

In particular, for $\alpha=4$ and $\beta=2$, Equation (41) is rewritten as

$$
\begin{aligned}
S_{\dot{1}}(\Gamma) & =1-\frac{2}{R_{\dot{1}}^{2}} \int_{0}^{R_{\mathrm{i}}} \frac{R \cdot e^{-\lambda C L \sqrt{\Gamma} R}}{\Gamma K R^{2}+1} \mathrm{~d} R \\
& =1-\frac{H\left(R_{\mathrm{i}}\right)}{R_{\mathrm{i}}^{2}}
\end{aligned}
$$

with

$$
\begin{aligned}
H(x)= & \int_{0}^{x} \frac{2 t e^{y t}}{z t^{2}+1} \mathrm{~d} t \stackrel{(a)}{=} \frac{1}{z}\left[e^{i y / \sqrt{z}}\{E(x y-i y / \sqrt{z})\right. \\
& -E(-i y / \sqrt{z})\}+e^{-i y / \sqrt{z}}\{E(x y+i y / \sqrt{z}) \\
& -E(i y / \sqrt{z})\}] \\
\stackrel{(b)}{=} & \frac{2}{z}[(\operatorname{Re}\{E(x y+i y / \sqrt{z})\}-\operatorname{Re}\{E(i y / \sqrt{z})\}) \\
& \times \cos (y / \sqrt{z})+(\operatorname{Im}\{E(x y+i y / \sqrt{z})\} \\
& -\operatorname{Im}\{E(i y / \sqrt{z})\}) \sin (y / \sqrt{z})],
\end{aligned}
$$

where $y=-\lambda C L \sqrt{\Gamma}, z=K \Gamma$. (a) follows from the integral formula in [33] and $(b)$ is given on the mirror symmetry of Exponential integral function. Combining Equations (42) and (43) gives the desired result (11).

Moreover, for the path loss exponents $\alpha=\beta=4$, by substitution $R^{2}=r$, Equation (41) is rewritten as

$$
S_{\mathrm{i}}(\Gamma)=1-\frac{1}{R_{\mathrm{i}}^{2}} \int_{0}^{R_{\mathrm{i}}^{2}} \frac{e^{-r \lambda C L \sqrt{\Gamma}}}{\Gamma K r^{2}+1} \mathrm{~d} r=1-\frac{B\left(R_{\mathrm{i}}^{2}\right)}{R_{\mathrm{i}}^{2}},
$$

where $B(x)$ is given from Equation (39). This gives the desired spatially averaged SIR distribution in Equation (13).

\section{Proof of Lemma 4}

The user SIR in (15) is rewritten as $\gamma(R)=\frac{g_{0}}{K\left(I_{1}+I_{2}\right)}$, where $K=\frac{P_{ \pm} D^{\alpha}}{P_{\mathrm{C}} L}$ and $I_{1}=h_{0} R^{-\beta}$ and $I_{2}=L^{2} \sum_{j \in \Phi \backslash A_{0}} h_{j}\left|X_{j}\right|^{-\alpha}$.
Using the same approach as in Equation (30), CCDF of the user SIR at distance $R$ from the FAP is given as

$$
\mathbb{P}[\gamma(R) \geq \Gamma]=\mathcal{L}_{I_{1}}(\Gamma K) \mathcal{L}_{I_{2}}(\Gamma K)=\frac{e^{-\lambda C\left(L^{2} K \Gamma\right)^{2 / \alpha}}}{\Gamma K R^{-\beta}+1}
$$

The home users connected to the central MBS are uniformly located at the zone $\mathcal{F}_{\mathrm{b}}$ that a circular annulus with outer radius $R_{\mathrm{i}}$ and inner radius $R_{\mathrm{f}}$. Then, PDF of $R$ is $f_{R}(r)=\frac{2 r}{R_{\mathrm{i}}^{2}-R_{\mathrm{f}}^{2}}, R \in\left[R_{\mathrm{f}}, R_{\mathrm{i}}\right]$. The spatially averaged SIR distribution over $\mathcal{F}_{\mathrm{b}}$ is given as

$$
\begin{aligned}
S_{\mathrm{b}}(\Gamma)= & \mathbb{E}_{R}[\mathbb{P}[\gamma(R) \leq \Gamma]]=1-\frac{2 e^{-\lambda C\left(L^{2} K \Gamma\right)^{2 / \alpha}}}{R_{\mathrm{i}}^{2}-R_{\mathrm{f}}^{2}} \\
& \int_{R_{\mathrm{f}}}^{R_{\mathrm{i}}} \frac{R}{\Gamma K R^{-\beta}+1} \mathrm{~d} R .
\end{aligned}
$$

Applying Equation (35) to (46) proves Equation (16).

\section{Proof of Theorem 1}

For a reference FAP at $D \leq D_{\text {th }}$, the home users in $\mathcal{F}_{a}$ connect to the FAP, while the remaining home users in $\mathcal{F}_{\mathrm{b}}$ communicate to the MBS. Thus, the average sum throughput of the home users is given as $T_{\mathrm{h}}^{\mathrm{CA}}=T_{1}+T_{2}$, where $T_{1}$ and $T_{2}$ are the average sum throughput of the home users in $\mathcal{F}_{\mathrm{a}}$ and $\mathcal{F}_{\mathrm{b}}$, respectively. Since the FAP supports the home users in $\mathcal{F}_{\mathrm{a}}$ only, we obtain $T_{1}=T_{\mathrm{a}}$, On the other hand, since the MBS transmits data to cellular users as well as the remaining $U_{\mathrm{b}}$ home users in $\mathcal{F}_{\mathrm{b}}, T_{2}$ is given as $T_{2}=\rho_{\mathrm{b}}^{\mathrm{CA}} T_{\mathrm{b}}$, and thus we get

$$
T_{\mathrm{h}}^{\mathrm{CA}}=T_{\mathrm{a}}+\rho_{\mathrm{b}}^{\mathrm{CA}} T_{\mathrm{b}}, \quad D \leq D_{\mathrm{th}} .
$$

Here, $\rho_{\mathrm{b}}^{\mathrm{CA}}$ is the fraction of time-slot dedicated to the $U_{\mathrm{b}}$ home users among all $\bar{U}_{\mathrm{m}}^{\mathrm{CA}}$ users supported by the MBS with TDMA and RR scheduling, which is given as

$$
\rho_{\mathrm{b}}^{\mathrm{CA}}=U_{\mathrm{b}} / \bar{U}_{\mathrm{m}}^{\mathrm{CA}}=U_{\mathrm{b}} /\left(U_{\mathrm{C}}+N_{\mathrm{f} 1} \bar{U}_{\mathrm{b}}\right),
$$

where $U_{\mathrm{b}}$ is given as

$$
U_{\mathrm{b}} \stackrel{(a)}{=} U_{\mathrm{h}}\left(1-\left(\frac{R_{\mathrm{f}}}{R_{\mathrm{i}}}\right)^{2}\right) \stackrel{(b)}{=} \pi \lambda_{\mathrm{h}}\left(R_{\mathrm{i}}^{2}-\kappa^{-2 / \alpha} D^{2}\right),
$$

where $(a)$ is given on the uniform distribution assumption of home users and (b) follows from Equation (3) and $U_{\mathrm{h}}=$ $\pi \lambda_{\mathrm{h}} R_{\mathrm{i}}^{2}$. In Equation (48), $\bar{U}_{\mathrm{b}}$ denotes the average number of users in $\mathcal{F}_{\mathrm{b}}$, which given as

$$
\begin{aligned}
\bar{U}_{\mathrm{b}} & =\mathbb{E}\left[U_{\mathrm{b}}\right]=\pi \lambda_{\mathrm{h}}\left(R_{\mathrm{i}}^{2}-\kappa^{-2 / \alpha} \mathbb{E}\left[D^{2}\right]\right) \\
& \stackrel{(a)}{=} \pi \lambda_{\mathrm{h}}\left(R_{\mathrm{i}}^{2}-\kappa^{-2 / \alpha} \frac{D_{\mathrm{th}}^{2}}{2}\right) \stackrel{(b)}{=} \frac{\pi \lambda_{\mathrm{h}} R_{\mathrm{i}}^{2}}{2},
\end{aligned}
$$

where (a) follows from $\mathbb{E}\left[D^{2}\right]=\int_{0}^{D_{\mathrm{th}}} D^{2}\left(\frac{2 D}{D_{\mathrm{th}}^{2}}\right) \mathrm{d} D=$ $D_{\text {th }}^{2} / 2$ for $D \leq D_{\text {th }}$ and (b) follows from $D_{\text {th }}=\kappa^{1 / \alpha} R_{\mathrm{i}}$. Moreover, $U_{C}$ is given as

$$
U_{\mathrm{C}}=\pi \lambda_{\mathrm{C}}\left(R_{\mathrm{C}}^{2}-N_{\mathrm{f}} R_{\mathrm{i}}^{2}\right) .
$$


Plugging Equations (49), (50), (51) into (48) gives the desired result in Equation (21).

Next, since a reference FAP with $D>D_{\text {th }}$ supports home users in the zone $\mathcal{F}_{i}$ only, the average sum throughput, $T_{\mathrm{h}}^{\mathrm{CA}}$, of the home users is equal to $T_{\mathrm{i}}$, which proves Equation (19). For a reference FAP at $D>D_{\text {th }}$, its neighboring cellular users in the zone $\mathcal{F}_{\circ}$ connect to the central MBS. Since the MBS transmits a data to some home users in inner region as well as $U_{\mathrm{C}}$ cellular users including the neighboring cellular users, the average sum throughput of the neighboring cellular users is given as

$$
T_{\mathrm{C}}^{\mathrm{CA}}=\rho_{\circ} T_{\circ}^{\mathrm{CA}},
$$

where $\rho_{\circ}$ is the fraction of time-slot dedicated to the $U_{\circ}$ neighboring cellular users in $\mathcal{F}_{\circ}$ among all $\bar{U}_{\mathrm{m}}^{\mathrm{CA}}$ users supported by the MBS with TDMA and RR scheduling, which is given as

$$
\rho_{\circ}=U_{\circ} / \bar{U}_{\mathrm{m}}^{\mathrm{CA}}=U_{\circ} /\left(U_{\mathrm{C}}+N_{\mathrm{f} 1} \bar{U}_{\mathrm{b}}\right),
$$

where $U_{\circ}$ is given as

$$
U_{\circ}=\pi \lambda_{\mathrm{C}}\left(R_{\mathrm{f}}^{2}-R_{\mathrm{i}}^{2}\right)=\pi \lambda_{\mathrm{C}}\left(\kappa^{-2 / \alpha} D^{2}-R_{\mathrm{i}}^{2}\right) .
$$

Plugging Equations (50), (51), (54) into (53) gives the desired result in Equation (22).

\section{Proof of Theorem 2}

In open access, for a reference FAP at $D \leq D_{\text {th }}$, the femtocell/macrocell access scenario of the home users in the zone $\mathcal{F}_{\mathrm{a}}$ and $\mathcal{F}_{\mathrm{b}}$ is the same as that in closed access. Thus, from Equation (47) the average sum throughput of the home users is thus given

$$
T_{\mathrm{h}}^{\mathrm{OA}}=T_{\mathrm{a}}+\rho_{\mathrm{b}}^{\mathrm{OA}} T_{\mathrm{b}}, \quad D \leq D_{\mathrm{th}}
$$

where $\rho_{\mathrm{b}}^{\mathrm{OA}}$ is is the fraction of time-slot dedicated to the $U_{\mathrm{b}}$ home users among all $\bar{U}_{\mathrm{m}}^{\mathrm{AA}}$ users supported by the MBS, which is given as

$$
\rho_{\mathrm{b}}^{\mathrm{OA}}=U_{\mathrm{b}} / \bar{U}_{\mathrm{m}}^{\mathrm{OA}}=U_{\mathrm{b}} /\left(U_{\mathrm{C}}-N_{\mathrm{f} 2} \bar{U}_{\mathrm{\circ}}+N_{\mathrm{f} 1} \bar{U}_{\mathrm{b}}\right)
$$

where $\bar{U}_{\mathrm{m}}^{\mathrm{OA}}=U_{\mathrm{C}}-N_{\mathrm{f} 2} \bar{U}_{\mathrm{\circ}}+N_{\mathrm{f} 1} \bar{U}_{\mathrm{b}}$ is the number of users served by the MBS in femtocell open access. Here, $N_{\mathrm{f} 2} \bar{U}_{\circ}$ is the number of cellular users accessing to the FAP $(D>$ $\left.D_{\mathrm{th}}\right)$ with open access. The average number of users in $\mathcal{F}_{\circ}, \bar{U}_{\circ}$, is given by

$$
\begin{gathered}
\bar{U}_{\circ}=\mathbb{E}\left[U_{\circ}\right] \stackrel{(a)}{=} \pi \lambda_{\mathrm{C}}\left(\kappa^{-2 / \alpha} \mathbb{E}\left[D^{2}\right]-R_{\mathrm{i}}^{2}\right) \\
\stackrel{(b)}{=} \pi \lambda_{\mathrm{C}}\left(\kappa^{-2 / \alpha} \frac{R_{\mathrm{c}}^{2}+D_{\mathrm{th}}^{2}}{2}-R_{\mathrm{i}}^{2}\right),
\end{gathered}
$$

where $(a)$ is given from Equation (54), and (b) follows from $\mathbb{E}\left[D^{2}\right]=\int_{D_{\mathrm{th}}}^{R_{\mathrm{c}}} D^{2}\left(\frac{2 D}{R_{\mathrm{c}}^{2}-D_{\mathrm{th}}^{2}}\right) \mathrm{d} D=\left(R_{\mathrm{C}}^{2}+D_{\mathrm{th}}^{2}\right) / 2$ for $D>D_{\text {th }}$. Plugging Equations (49), (50), (51), (57) into (56) gives the desired result in Equation (25).

For a reference FAP at $D>D_{\text {th }}$, since the MBS transmits a data to the neighboring cellular users in $\mathcal{F}_{\circ}$ as well as the home users in $\mathcal{F}_{i}$, the average sum throughput of the home users is given as

$$
T_{\mathrm{h}}^{\mathrm{OA}}=\rho_{\mathrm{i}} T_{\mathrm{i}}, T_{\mathrm{C}}^{\mathrm{OA}}=\left(1-\rho_{\mathrm{i}}\right) T_{\mathrm{O}}^{\mathrm{OA}},
$$

where $\rho_{\mathrm{i}}$ is the fraction of time slot dedicated to the home users in $\mathcal{F}_{i}$ among home and cellular users supported from the FAP, which is given as

$$
\rho_{\mathrm{i}}=U_{\mathrm{i}} /\left(U_{\mathrm{i}}+U_{\circ}\right)
$$

Plugging Equations (54) and $U_{\mathrm{i}}=U_{\mathrm{h}}=\pi \lambda_{\mathrm{h}} R_{\mathrm{i}}^{2}$ into (59) gives the desired result in Equation (26).

\section{Endnotes}

${ }^{\text {a }}$ Fixed power (no transmit power control of femtocells) is assumed as in [16,18-21], to focus on the effects of femtocell access scheme.

${ }^{\mathrm{b}}$ Figure 3 suggests that the assumption is not a significant omission of interference effects for dense femtocell systems. See Section 3.3 for more discussion.

${ }^{\mathrm{c}} \mathrm{A}$ similar approach is presented in [10], which considers different path loss exponents as well as the same path loss exponents for macrocell and femtocell, but not includes wall penetration loss.

\section{Competing interests}

The authors declare that they have no competing interests.

\section{Acknowledgements}

This study was supported by the KCC (Korea Communications Commission), Korea, under the R\&D program supervised by the KCA (Korea Communications Agency) (KCA-2012-12-911-01-108) and Motorola Solutions. This article was partially presented at the International Conference on Communications, Kyoto, Japan, June 5-9, 2011 [34].

\section{Author details}

${ }^{1}$ Department of Electronics and Control Engineering, National Hanbat University, Daejeon, Korea. ${ }^{2}$ Department of Electrical and Computer Engineering, The University of Texas at Austin, Austin, TX, USA.

Received: 21 November 2011 Accepted: 14 November 2012 Published: 15 December 2012

\section{References}

1. P Xia, HS Jo, J Andrews, Fundamentals of inter-cell overhead signaling in heterogeneous cellular networks. IEEE J. Sel. Topics Signal Process. 6(3), 257-269 (2012)

2. V Chandrasekhar, JG Andrews, A Gatherer, Femtocell networks: a Survey. IEEE Commun. Mag. 46(9), 59-67 (2008)

3. A Damnjanovic, J Montojo, Y Wei, T Ji, T Luo, M Vajapeyam, T Yoo, O Song, D Malladi, A survey on 3GPP heterogeneous networks. IEEE Wirel. Commun. 18(3), 10-21 (2011)

4. Y Li, A Maeder, L Fan, A Nigam, J Chou, Overview of femtocell support in advanced WiMAX systems. IEEE Commun. Mag. 49(7), 122-130 (2011)

5. P Mach, Z Becvar, QoS-guaranteed power control mechanism based on the frame utilization for femtocells. EURASIP J. Wirel. Commun. Netw. 2011(Article ID 802548), 16 (2011)

6. M Yavuz, F Meshkati, S Nanda, A Pokhariyal, N Johnson, B Raghothaman, A Richardson, Interference management and performance analysis of UMTS/HSPA+ femtocells. IEEE Commun. Mag. 47(9), 102-109 (2009)

7. HS Jo, C Mun, J Moon, JG Yook, Interference mitigation using uplink power control for two-tier femtocell networks. IEEE Trans. Wirel. Commun. 8(10), 4906-4910 (2009) 
8. HS Jo, C Mun, J Moon, JG Yook, Self-optimized coverage coordination in femtocell networks. IEEE Trans. Wirel. Commun. 9(10), 2977-2982 (2010)

9. V Chandrasekhar, M Kountouris, JG Andrews, Coverage in multi-antenna two-tier networks. IEEE Trans. Wirel. Commun. 8(10), 5314-5327 (2009)

10. I Guvenc, MR Jeong, F Watanabe, H Inamura, A hybrid frequency assignment for femtocells and coverage area analysis for co-channel operation. IEEE Commun. Lett. 12(12), 880-882 (2008)

11. D Lopez-Perez, A Ladanyi, A Juttner, J Zhang, in IEEE 20th International Symposium on Personal, Indoor and Mobile Radio Communications, 2009. OFDMA femtocells: a self-organizing approach for frequency assignment, (Tokyo, Japan, 2009), pp. 2202-2207

12. V Chandrasekhar, JG Andrews, Spectrum allocation in two-tier networks. IEEE Trans. Commun. 57(10), 3059-3068 (2009)

13. JPM Torregoza, R Enkhbat, WJ Hwang, Joint power control, base station assignment, and channel assignment in cognitive femtocell networks. EURASIP J. Wirel. Commun. Netw. 2010, 6:1-6:12 (2010)

14. S Al-Rubaye, A Al-Dulaimi, J Cosmas, Cognitive femtocell. IEEE Veh. Technol. Mag. 6, 44-51 (2011)

15. H Claussen, in IEEE 18th International Symposium on Personal, Indoor and Mobile Radio Communications, 2007, PIMRC 2007. Performance of macroand co-channel femtocells in a hierarchical cell structure (Athens, Greece, 2007), pp. 1-5

16. S Joshi, R Cheung, P Monajemi, J Villasenor. Traffic-based study of femtocell access policy impacts on HSPA service quality (Honolulu, USA, 2009), pp. 1-6

17. P Xia, V Chandrasekhar, J Andrews, Open vs. closed access femtocells in the uplink. IEEE Trans. Wirel. Commun. 9(12), 3798-3809 (2010)

18. D Choi, P Monajemi, S Kang, J Villasenor, in IEEE Global Telecommunications Conference, 2008. Dealing with loud neighbors: the benefits and tradeoffs of adaptive femtocell access (New Orleans, USA, 2008), pp. 1-5

19. M Simsek, H Wu, B Zhao, T Akbudak, A Czylwik, in Wireless Advanced (WiAd), 2011. Performance of different cell selection modes in 3GPP-LTE macro-/femtocell scenarios (London, UK, 2011), pp. 126-131

20. G de la Roche, A Valcarce, D Lopez-Perez, J Zhang, Access control mechanisms for femtocells. IEEE Commun. Mag. 48, 33-39 (2010)

21. A Valcarce, D Lopez-Perez, G De La Roche, J Zhang, in IEEE 20th International Symposium on Personal, Indoor and Mobile Radio Communications, 2009. Limited access to OFDMA femtocells (Tokyo, Japan, 2009), pp. 1-5

22. HS Dhillon, RK Ganti, F Baccelli, JG Andrews, Modeling and analysis of K-tier downlink heterogeneous cellular networks. IEEE J. Sel. Areas Commun. 30(3), 550-560 (2012)

23. HS Jo, YJ Sang, P Xia, J Andrews, in IEEE Global Telecommunications Conference (GLOBECOM 2011), 2011. Outage probability for heterogeneous cellular networks with biased cell association (Houston, USA, 2011), pp. 1-5

24. HS Jo, YJ Sang, P Xia, JG Andrews, Heterogeneous cellular networks with flexible cell association: a comprehensive downlink SINR analysis. IEEE Trans. Wirel. Commun. 11(10), 3484-3495 (2012)

25. S Mukherjee, in IEEE International Conference on Communications (ICC), 2011. Analysis of UE outage probability and macrocellular traffic offloading for WCDMA macro network with femto overlay under closed and open access (Kyoto, Japan, 2011), pp. 1-6

26. M Haenggi, JG Andrews, F Baccelli, O Dousse, M Franceschetti, Stochastic geometry and random graphs for the analysis and design of wireless networks. IEEE J. Sel. Areas Commun. 27(7), 1029-1046 (2009)

27. JG Andrews, RK Ganti, N Jindal, M Haenggi, S Weber, A primer on spatial modeling and analysis in wireless networks. IEEE Commun. Mag. 48(11), 156-163 (2010)

28. Y Kim, S Lee, D Hong, Performance analysis of two-tier femtocell networks with outage constraints. IEEE Trans. Wirel. Commun. 9(9), 2695-2700 (2010)

29. 3GPP TS 25.104 V8.10.0, Base Station radio transmission and reception (FDD) (2010)

30. MS Alouini, A Goldsmith, Area spectral efficiency of cellular mobile radio systems. IEEE Trans. Veh. Technol. 48(4), 1047-1066 (1999)

31. F Baccelli, B Blaszczyszyn, P Muhlethaler, An ALOHA protocol for multihop mobile wireless networks. IEEE Trans. Inf. Theory. 8(6), 569-586 (2006)
32. A Jeffrey, D Zwillinger, Tables of Integrals, Series, and Products. (Academic Press, New York, 2007)

33. http://functions.wolfram.com/ElementaryFunctions/Exp/21/01/02/

34. HS Jo, P Xia, J Andrews, in IEEE International Conference on Communications (ICC), 2011. Downlink femtocell networks: open or closed? (Kyoto, Japan, 2011), pp. 1-5

doi:10.1186/1687-1499-2012-363

Cite this article as: Jo et al:: Open, closed, and shared access femtocells in the downlink. EURASIP Journal on Wireless Communications and Networking 2012 2012:363.

\section{Submit your manuscript to a SpringerOpen ${ }^{\mathcal{O}}$ journal and benefit from:}

- Convenient online submission

- Rigorous peer review

- Immediate publication on acceptance

- Open access: articles freely available online

- High visibility within the field

- Retaining the copyright to your article

Submit your next manuscript at $>$ springeropen.com 